\title{
The Order of the Molecular Chains in Isotactic Polypropylene Crystals
}

\author{
Masamichi Hikosaka and Tsuneo Seto \\ Department of Physics, Faculty of Science, \\ Tokyo Metropolitan University, \\ Setagaya-ku, Tokyo, Japan.
}

(Received September, 18, 1972)

\begin{abstract}
When isotactic polypropylene $\alpha$ form was annealed at high temperatures, a structural transition from a disordered state to an ordered state was observed and studied by X-ray diffraction. The space groups of the two forms were proved to be $\mathrm{C} 2 / \mathrm{c}$ and $\mathrm{P} 2_{1} / \mathrm{c}$ respectively. We proposed an "order domain eduction model" for the transition, from an analysis of reflected intensity and line width. The structure factor of the intermediate state was then written

$$
F_{\text {int }}=\bar{n} F_{\mathrm{C} 2 / \mathrm{c}}+(1-\bar{n}) F_{\mathrm{P}_{1} / \mathrm{c}}
$$

where $\bar{n}$ is the volume fraction of the $\mathrm{C} 2 / \mathrm{c}$ form; this gave a good value of the $R$ factor, 0.152. From structural considerations, the transition was interpreted as the arrangement of $\mathrm{CH}-\mathrm{CH}_{3}$ bonds (upward or downward) changing into complete order, where the helical arrangement was already in complete order in both forms; reasons for this model were discussed using energy considerations. The origins of streak-like diffuse scattering and of various disorders in the crystal were also discussed.

KEY WORDS Isotactic Polypropylene / X-ray Diffraction / Disorder-Order Transition / Structural Analysis / Line Width / Diffuse Scattering (Streak) /
\end{abstract}

The structural analysis of isotactic polypropylene (ipp) $\alpha$ form crystal has been proposed independently by Natta and Mencik; they suggest a mixture of $\mathrm{C} 2 / \mathrm{c}$ and $\mathrm{Cc}$ and $\mathrm{P}_{1} / \mathrm{c}$ space groups. ${ }^{1,2}$ There has still remained the problem of deciding which space group should be taken. We give a confirmative structural model, on the basis of our experimental results, for a disorder-order transition in ipp $\alpha$ form crystal. In this paper it is shown that the disorderorder transition is related to an ordering of the molecular chain arrangement. Our study was carried out by using X-ray diffraction. We first report on the experimental fact that two different phases are obtained when the annealing temperature is raised, and give the results of structural analysis of the two phases. We then propose a model for the transition mechanism by combining the analysis results with the observed data of the intensity and the line width. We discuss four points: the relation between our crystal structure and that proposed by Natta or Mencik, a consideration of the transition from an energy standpoint, our interpretation of the streak-like diffuse scattering, and relations between our disorder state and the other disorder states of ipp $\alpha$ form.

\section{EXPERIMENTAL}

The isotactic polypropylene used was Sumitomo Noblene (D501) with an average molecular weight of 250,000. Three types of specimens were prepared. (1) Unoriented specimen; plates about $1-\mathrm{mm}$ thick were melted, quenched in ice water, and annealed at various temperatures between $120-170^{\circ} \mathrm{C}$ in an oil bath for $1-2 \mathrm{hr}$. (2) Uniaxially oriented specimen; pellets were melted and made into a plate by quenching in ice water. They were drawn to about $400-700 \%$ at room temperature through the necking stage. Annealing was carried out at various temperatures between $120-170^{\circ} \mathrm{C}$ for $10 \mathrm{~min}-113 \mathrm{hr}$ under either an isometric condition or a semi- 
atonic condition. During the annealing, the specimens were shortened to about $3 / 4$ of their previous length. 3) Solution-grown crystals; isothermal crystallization was performed from dilute solution. The polymer was dissolved in the solvent, $\alpha$-chlornaphthalene, to 0.02 wt $\%$ at about $200^{\circ} \mathrm{C}$ and the solution was brought into an oil bath and crystallized at $106^{\circ} \mathrm{C}$. The floating crystals were filtered and dried in vacuum. All these specimens were examined by the $\mathrm{X}$ ray method with $\mathrm{Ni}$-filtered $\mathrm{CuK} \alpha$ radiation at room temperature. Lattice constants were obtained using a cylindrical camera of $69.52 \mathrm{~mm}$ in radius, with copper powder as a reference material. Intensity measurements were carried out by a Weissenberg camera on the uniaxially oriented specimens by the multifilms method, and the line profiles were observed by a microphotometer.

\section{RESULTS}

A drastic change was found in the X-ray diffraction pattern when the annealing temperature was raised, as is shown in Figure 1, (a)-(b) and (c)-(d). In order to analyze two different states, specimens A and B were prepared.

$\left\{\begin{aligned} \text { Specimen A: drawn to } 700 \% \text { and annealed } & \\ \text { under isometric conditions for } & 2 \mathrm{hr} \text { at } 140^{\circ} \mathrm{C} . \\ \text { Specimen B: } & \text { drawn to } 700 \% \text { and annealed } \\ \text { under isometric conditions for } & 2 \mathrm{hr} \text { at } 171^{\circ} \mathrm{C} \text {. }\end{aligned}\right.$

Both crystal lattices belonged to the monoclinic system which was characteristic of $\alpha$ form.

Lattice constants and reflection indicies were refined by a self-consistent method starting from Natta's lattice constants. The lattice constants were

for specimen $\mathrm{A}: \begin{array}{r}a=6.67 \AA, b=20.8 \AA, c= \\ 6.50 \AA \text {, and } \beta=98^{\circ} 40^{\prime} \text { (at } \\ 25^{\circ} \mathrm{C} \text { ) } \\ \text { for specimen B: } a=6.65 \AA, b=20.73 \AA, c= \\ 6.50 \AA, \text { and } \beta=98^{\circ} 40^{\prime} \text { (at } \\ 25^{\circ} \mathrm{C} \text { ). }\end{array}$
The reflections $h k l$ with $h+k=$ odd, for exThe reflections $h k l$ with $h+k=$ odd, for example, $290, \frac{1120}{380}, 141, \frac{\overline{2} 31}{116}, 211, \overline{2} 51, \frac{\overline{1} 22}{032}, \overline{1} 422$, $\overline{1} 62, \frac{\overline{2} 13}{123}$, etc., were systematically absent in specimen A, but they began to appear and in-

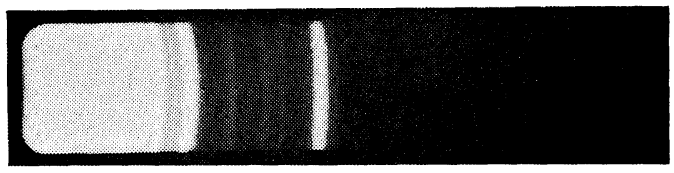

(a)

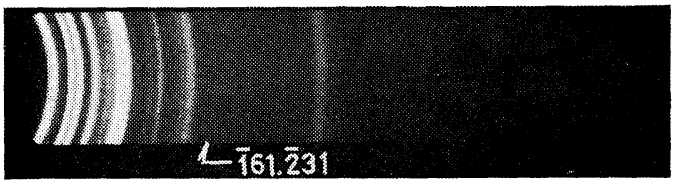

(b)

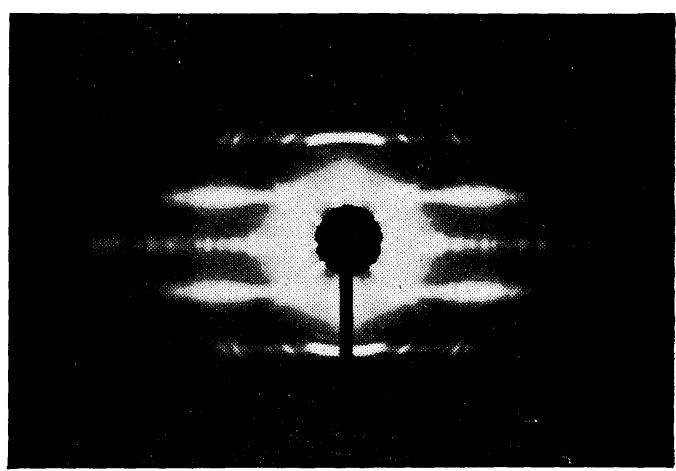

(c)

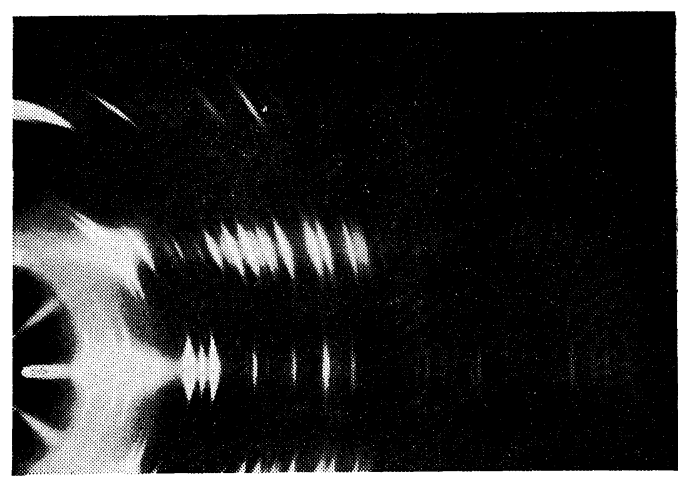

(d)

Figure 1. X-ray diagrams. Unoriented and annealed: (a) at $115^{\circ} \mathrm{C}$ for $2 \mathrm{hr}$, (b) at $165^{\circ} \mathrm{C}$ for $2 \mathrm{hr}$. Drawn to $700 \%$ and annealed for $2 \mathrm{hr}$ under isometric conditions: (c) at $140^{\circ} \mathrm{C}$, (d) at $171^{\circ} \mathrm{C}$.

creased in intensity as the annealing temperature was raised. The variation of intensity of one of the most typical reflections, $\overline{\mathbf{2}} 31$, is illustrated in Figure 2. We name the form " $\alpha 1$ form" in which the reflections $h k l$ with $h+k=$ odd are 
extinct, and the form " $\alpha 2$ form" in which the intensity of the same reflections reaches the maximum value. We also name the state in the middle of the transition the "intermediate form." It is shown in Figure 2 that the $\alpha 1$

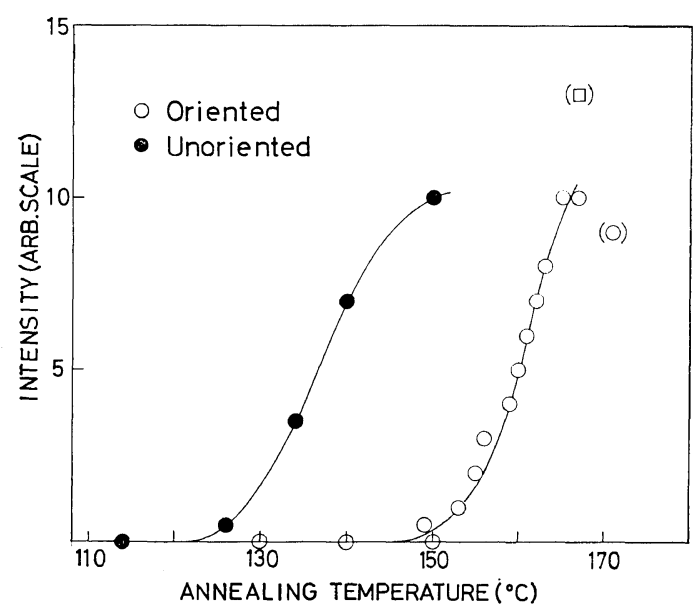

Figure 2. Change in the diffraction intensity of 23161: - undrawn; $\bigcirc$, drawn to $700 \%$, annealed under fixed conditions; ( $\square$ ), drawn to $600 \%$, annealed under semi-atonic conditions; $(O)$, B specimen.

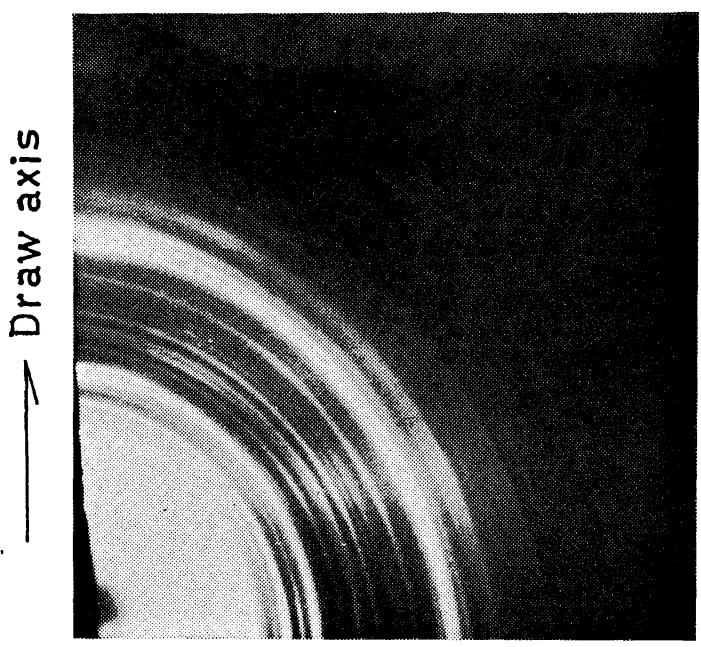

Figure 3. X-ray pattern. Drawn to $600 \%$ and annealed at $169^{\circ} \mathrm{C}$ for $30 \mathrm{~min}$ under semi-atonic conditions. Photographed by equi-inclination method, $\mu=20^{\circ} 51^{\prime}$. form transforms to the intermediate form on an S-shaped curve. The state close to the $\alpha 2$ form, however, is obtained only by drawing to $600 \%$ and annealing under semiatonic condition at $167^{\circ} \mathrm{C}$, which is shown by ( $\square$ ) in Figure 2, or some similar treatment. The X-ray pattern of the state close to the $\alpha 2$ form is shown in Figure 3 , in which the $c$-axis orientation is very broad. The specimens $\mathrm{A}$ and $\mathrm{B}$ correspond to the $\alpha 1$ and the intermediate form respectively.

Once the $\alpha 1$ form transforms into the $\alpha 2$ form, the process is not reversed when the material is cooled to room temperature. The transition depends on the treatment of the specimens, in particular on the drawing ratio. The transition temperature becomes lower as the drawing ratio becomes smaller. In the X-ray pattern of the solution-grown specimen, the reflection $\frac{\overline{2} 31}{161}$ is clearly observed; thus it belongs to the intermediate or $\alpha 2$ form. The line width of $h k l$ with $h+k=$ odd was always broader than that of $h k l$ with $h+k=$ even in the intermediate form. One example is illustrated in Figure 4. The line profile of the intermediate form is not very deformed until a large scattering angle is reached, hence it is reasonable to assume that

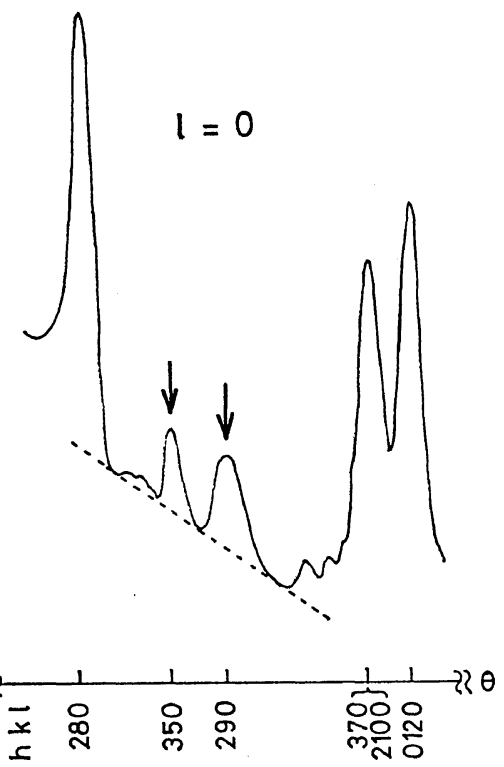

Figure 4. Line profile of a part of the equatorial line. 
the line width is due mainly to the size of a crystallite. The size of a crystallite lateral to the $c$-axis was obtained by using two reflections, 350 and 290, assuming a Gaussian profile, and using Scherrer's and Stokes' method ${ }^{3}$; the result is illustrated in Figure 5.

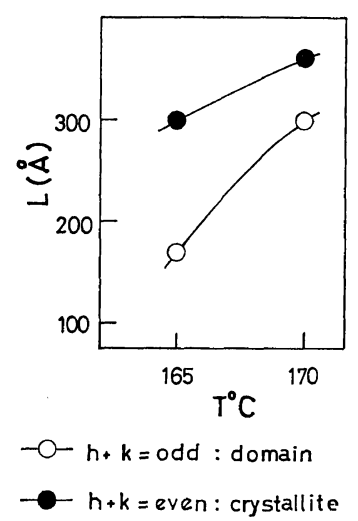

Figure 5. Size of crystallite and domain obtained from line widths of 350 and 290 respectively.

An intensity measurement of the A and B specimens was carried out, which will be given in detail in the ensuing section.

\section{CRYSTAL STRUCTURES}

Structural analysis of the $\alpha 1$ and the intermediate form was carried out. The X-ray patterns of both forms are similar to those of Natta, as far as the reflections $h k l$ with $h+k=$ even are concerned, and therefore it is appropriate to start the analysis from Natta's model. ${ }^{1}$

\section{Structure of the Intermediate Form}

The lattice should be a primitive one, because of the existence of the new Bragg reflection $h k l$ with $h+k=$ odd. The reflections $0 k 0$ with $k=$ odd, 090, 0130, 0170, etc., and the reflections $h 0 l$ with $l$ or $h=$ odd, 201 and 401 or 102 and 104 , are systematically absent; thus, from the extinction rule, the space group of the $\alpha 2$ form should be either $\mathbf{P} 2_{1} / \mathrm{c}$ or $\mathrm{P} 2_{1} / \mathrm{a}$. The $\mathrm{P} 2_{1} / \mathrm{c}$ space group is selected for the reason that the structure factors calculated from the $\mathrm{P} 2_{1} / \mathrm{c}$ space group agree with those observed better than those from the other space group. The agreement is good in the equatorial line, but in the other lines there are some deviations, which increase as the $l$ in $h k l$ goes higher. This suggests that the $z$ atomic coordinates are inappropriate, and hence the $z$ coordinates are altered in terms of $\Delta z$

$$
z=z_{0}+\frac{\Delta z}{360}
$$

where $z_{0}$ and $z$ are Natta's and the altered $z$ coordinates respectively. We define another packing parameter $\theta$, which measures the rotation of a chain around its chain axis (see Figure 6). Chain shape is altered by use of bond angle

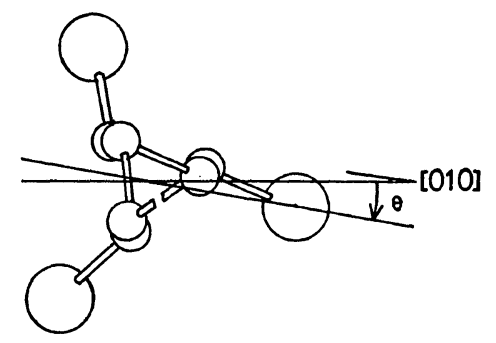

Figure 6. Definition of a chain-settlement parameter $\theta$ in the (001) projection.

parameters. These parameters were determined by trial and error so as to minimize the $R$ factor, which defined as follows:

$$
R=\frac{\sum\left|F_{\mathrm{d}}\right|}{\sum\left|F_{\mathrm{c}}\right|}
$$

where $F_{\mathrm{d}}=\left|F_{0}\right|-\left|F_{\mathrm{c}}\right|, F_{0}$ and $F_{\mathrm{c}}$ are the observed and the calculated structure factors, and the summation is carried out over all the sets of $h k l$.

It is reasonable to consider that the intermediate form is composed of the $\alpha 1$ and $\alpha 2$ forms, and that the space group of the $\alpha 2$ form is $\mathbf{P} 2_{1} /$ c. As shown later, the structure factor of the intermediate form $F_{\text {int }}$ is given as

$$
F_{\text {int }}=\bar{n} F_{\alpha 1}+(1-\bar{n}) F_{\alpha 2}
$$

where $F_{\alpha 1}$ and $F_{\alpha 2}$ are the structure factors of the $\alpha 1$ and $\alpha 2$ forms and $\bar{n}$ is the volume fraction of the $\alpha 1$ form. We have already seen

where

$$
F_{\alpha 2}=F_{\mathrm{P}_{2} / \mathrm{c}}
$$

$$
\begin{gathered}
F_{\mathrm{P} 2_{3} / \mathrm{c}}=\sum_{j} \\
4 f_{\mathrm{c}} \cos 2 \pi\left(h x_{j}+l z_{j}+\frac{k+l}{4}\right) \\
\times \cos 2 \pi\left(k y_{j}-\frac{k+l}{4}\right)
\end{gathered}
$$


in which $f_{\mathrm{c}}$ is the atomic structure factor of carbon.

We will show in the following section

where

$$
F_{\alpha 1}=F_{\mathrm{C} 2 / \mathrm{c}}
$$

$$
\begin{aligned}
F_{\mathrm{C} 2 / \mathrm{c}}= & \sum_{j} \frac{8}{2} f_{\mathrm{c}} \cos ^{2} 2 \pi \frac{h+k}{4} \\
& \times \cos 2 \pi\left(h x_{j}+l z_{j}+\frac{1}{4}\right) \\
& \times \cos 2 \pi\left(k y_{j}-\frac{1}{4}\right)
\end{aligned}
$$

Inserting eq 4 and 6 into eq 3 , we have

$$
F_{\text {int }}=\bar{n} F_{\mathrm{C} 2 / \mathrm{c}}+(1-\bar{n}) F_{\mathrm{P} 2_{1} / \mathrm{c}}
$$

A detailed proof for eq (8) will be given in the ensuing section.

Taking $F_{\mathrm{c}}=F_{\text {int }}$ in eq (2), the best value of the $R$-factor $(R=0.152)$ is obtained when we take the following chain shape, packing parameters, and vloume fraction, as shown in Figure 7:

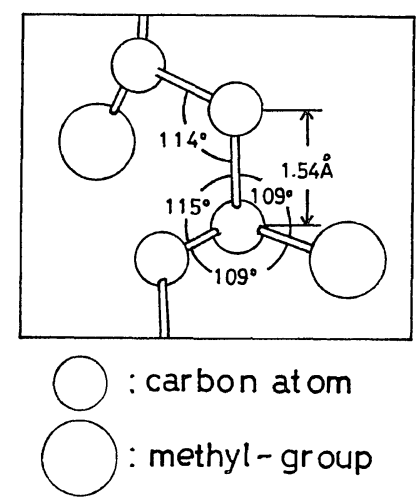

Figure 7. The chain shape assumed by us.

chain shape: $\mathrm{C}-\mathrm{C}$ bond length is $1.54 \AA$ and the bond angles $\mathrm{CH}_{2}-$ $\mathrm{CH}-\mathrm{CH}_{2}, \mathrm{CH}-\mathrm{CH}_{2}-\mathrm{CH}$, and $\mathrm{CH}_{2}-\mathrm{CH}-\mathrm{CH}_{3}$ are $114^{\circ}, 115^{\circ}$, and $109^{\circ}$, as illustrated in Figure 7;

packing parameters: $(\theta, \Delta z)=(0,24)$;

volume fraction: $\bar{n}=0.66$.

The $\Delta z$ and $\tilde{n}$ dependences of the $R$-factor are indicated in Figures $8 \mathrm{a}$ and $\mathrm{b}$.

The final atomic coordinates and structure factors are listed in Tables Ia and b. The $R$ value of 0.152 is sufficiently accurate. The unit

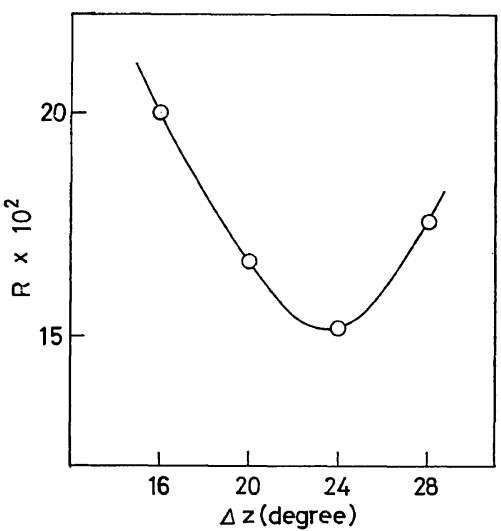

(a)

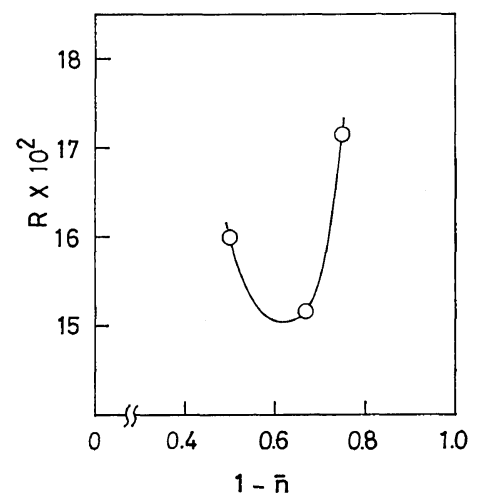

(b)

Figure 8. $R$-factor $v s$. (a) $\Delta z$, where $\theta=0$; (b) volume fraction of the $\alpha 2$ form, $(1-\bar{n})$, where $(\theta$, $\Delta z)=(0,24)$.

Table Ia. Coordinates of the independent structural unit of polypropylene used in calculating structure factors of both the intermediate form and the $\alpha 1$ form, neglecting hydrogen atoms. The chain shape illustrated in Figure 8 and the following packing parameter values are taken: $(\theta, \Delta z)=(0,24)$.

\begin{tabular}{crrc}
\hline \multirow{2}{*}{ Carbon atoms } & \multicolumn{3}{c}{ Coordinates of the carbon atoms } \\
\cline { 2 - 4 } & \multicolumn{1}{c}{$x$} & $y$ & $z$ \\
\hline $\mathrm{C}_{1}$ & -0.0423 & -0.1759 & 0.4699 \\
$\mathrm{C}_{2}$ & 0.1669 & -0.1527 & 0.5805 \\
$\mathrm{C}_{3}$ & 0.1680 & -0.1478 & 0.8171 \\
$\mathrm{C}_{4}$ & 0.5423 & -0.1759 & 0.8968 \\
$\mathrm{C}_{5}$ & 0.3744 & -0.1300 & 0.9470 \\
$\mathrm{C}_{6}$ & 0.3606 & -0.1321 & 0.1813 \\
$\mathrm{C}_{7}$ & 0.2500 & -0.0152 & 0.1833 \\
$\mathrm{C}_{8}$ & 0.2087 & -0.0843 & 0.2538 \\
$\mathrm{C}_{9}$ & 0.2214 & -0.0870 & 0.4923 \\
\hline
\end{tabular}


Table Ib. Comparison between the observed and calculated structure factors of the ipp intermediate form. The $F_{0}$ values are obtained after correcting for the usual angular factors. The temperature factor $B=8.0$. In order to take into account the different mutliplicities of the reflections, the $F(h k l)$ were multiplied by the following $M ; M=1$ for $h 0 l, M=2$ for $h k 0$, and $M=\sqrt{2}$ for the others.

\begin{tabular}{|c|c|c|c|c|c|c|c|c|c|c|c|c|c|}
\hline$h$ & $k$ & $l$ & $d^{-1} \AA^{\mathrm{a}}$ & $\left|F_{0}\right|^{\mathrm{a}}$ & $\left|F_{\text {int }}\right|^{b}$ & $F_{\mathrm{d}^{\mathrm{e}}}$ & $h$ & $k$ & $l$ & $d^{-1} \AA$ & $\left|F_{0}\right|$ & $\left|F_{\text {int }}\right|$ & $F_{\mathrm{d}}$ \\
\hline 0 & 2 & 0 & - & - & 1.2 & 1.2 & 1 & 14 & 0 ) & 0.697 & 8.6 & 14.2 & -5.6 \\
\hline 1 & 0 & 0 & & & 1.6 & 1.6 & 4 & 7 & 0 & & & & \\
\hline 1 & 1 & 0 & 0.160 & 140.4 & 128.0 & 12.4 & $\begin{array}{l}2 \\
3\end{array}$ & $\begin{array}{l}13 \\
11\end{array}$ & $\begin{array}{l}0 \\
0\end{array}$ & & & & \\
\hline 1 & 2 & 0 & - & - & 0.0 & 0.0 & 4 & 8 & 0 & 0.721 & 14.2 & 16.8 & -2.6 \\
\hline 0 & 4 & 0 & 0.193 & 119.2 & 118.0 & 1.2 & 3 & 12 & 0 ) & 0.740 & 30.4 & 33.4 & -3.0 \\
\hline 1 & 3 & 0 & 0.210 & 148.0 & 140.2 & 7.8 & 2 & 14 & $\begin{array}{l}0 \\
0\end{array}$ & & & & \\
\hline 1 & 4 & 0 & - & - & 6.4 & 6.4 & 1 & 15 & $0)$ & & & & \\
\hline 1 & 5 & $0\}$ & 0.289 & 78.8 & 71.8 & 7.0 & 4 & 9 & 0 & - & - & 1.6 & 1.6 \\
\hline 0 & 6 & 0 & & & & & 5 & 0 & 0 & 0.762 & 21.4 & 22.0 & -0.6 \\
\hline $\begin{array}{l}2 \\
2\end{array}$ & $\begin{array}{l}0 \\
1\end{array}$ & $\left.\begin{array}{l}0 \\
0\end{array}\right\}$ & 0.305 & 41.0 & 47.6 & -6.6 & $\begin{array}{l}5 \\
5\end{array}$ & $\begin{array}{l}1 \\
2\end{array}$ & $\left.\begin{array}{l}0 \\
0\end{array}\right\}$ & & & & \\
\hline 2 & 2 & 0 & 0.319 & 76.6 & 70.4 & 6.2 & 0 & 16 & $\left.\begin{array}{l}0 \\
0\end{array}\right)$ & 0.776 & 23.8 & 27.2 & -3.4 \\
\hline 1 & 6 & 0 & - & - & 1.2 & 1.2 & $\begin{array}{l}5 \\
4\end{array}$ & $\begin{array}{r}3 \\
10\end{array}$ & $\begin{array}{l}0 \\
0\end{array}$ & & & & \\
\hline 2 & 3 & 0 & - & - & 2.4 & 2.4 & 3 & 13 & $0)$ & & & & \\
\hline 2 & 4 & 0 & 0.360 & 10.4 & 14.0 & -3.6 & 1 & 16 & 0 ) & - & - & 14.4 & 14.4 \\
\hline 1 & 7 & 0 & 0.370 & 31.0 & 28.8 & 2.2 & 2 & 15 & $0\}$ & & & & \\
\hline 0 & 8 & $\left.\begin{array}{l}0 \\
0\end{array}\right\}$ & 0.387 & 10.8 & 12.8 & -2.0 & $\begin{array}{l}5 \\
5\end{array}$ & $\begin{array}{l}4 \\
5\end{array}$ & $\begin{array}{l}0) \\
0\end{array}$ & 0.798 & 238 & 236 & 0.2 \\
\hline $\begin{array}{l}2 \\
1\end{array}$ & $\begin{array}{l}3 \\
8\end{array}$ & 0 & 0.419 & 29.4 & 37.0 & -7.6 & 4 & 11 & 0 & 0.811 & 7.0 & 6.0 & 1.0 \\
\hline 2 & $\begin{array}{l}0 \\
6\end{array}$ & 0 & & & & -1.0 & 5 & 6 & 0 & - & - & 3.8 & 3.8 \\
\hline 2 & 7 & 0 & 0.459 & 38.4 & 42.4 & -4.0 & 3 & 14 & 0 & & & & \\
\hline 3 & 0 & 0 & & & & & 2 & 16 & 0 & 0.830 & 14.4 & 21.2 & -6.8 \\
\hline 3 & 1 & 0 & & & & & $\begin{array}{l}5 \\
1\end{array}$ & $\begin{array}{r}7 \\
17\end{array}$ & $\begin{array}{l}0 \\
0\end{array}$ & & & & \\
\hline 1 & 9 & 0 & & & & & 4 & 12 & 0 & & & & \\
\hline 3 & 2 & 0 & - & - & 1.0 & 1.0 & 5 & 8 & 0 & - & - & 1.4 & 1.4 \\
\hline 0 & 10 & 0 & 0.475 & 6.0 & 15.8 & -9.8 & 3 & 15 & 0 & 0.862 & 9.2 & 12.4 & -3.2 \\
\hline $\begin{array}{l}2 \\
3\end{array}$ & $\begin{array}{l}8 \\
4\end{array}$ & $\left.\begin{array}{l}0 \\
0\end{array}\right\}$ & 0.493 & 28.0 & 30.2 & -2.2 & $\begin{array}{l}0 \\
4\end{array}$ & $\begin{array}{l}18 \\
13\end{array}$ & $\begin{array}{l}0 \\
0\end{array}$ & & & & \\
\hline 1 & 10 & 0 & - & - & 2.1 & 2.1 & $\begin{array}{l}2 \\
5\end{array}$ & $\begin{array}{r}17 \\
9\end{array}$ & $\begin{array}{l}0 \\
0\end{array}$ & & & & \\
\hline 3 & 5 & 0 & 0.516 & 10.2 & 15.4 & -5.2 & 0 & 1 & 1 & - & - & 2.2 & 2.2 \\
\hline 2 & 9 & 0 & 0.533 & 10.4 & 11.0 & -0.6 & 0 & 2 & 1 & - & - & 0.0 & 0.0 \\
\hline 3 & 6 & 0 & - & - & 0.4 & 0.4 & -1 & 1 & 1 & 0.206 & 8.3 & 7.1 & 1.2 \\
\hline 1 & 11 & 0 & - & - & 6.0 & 6.0 & 0 & 3 & 1 & - & - & 0.7 & 0.7 \\
\hline $\begin{array}{l}3 \\
2\end{array}$ & $\begin{array}{r}7 \\
10\end{array}$ & $\left.\begin{array}{l}0 \\
0\end{array}\right\}$ & 0.569 & 21.2 & 20.6 & 0.6 & -1 & 2 & 1 & - & - & 0.1 & 0.1 \\
\hline 0 & 12 & 0 & 0.579 & 23.4 & 21.2 & 2.2 & 1 & 1 & 1 & 0.238 & 78.2 & 76.5 & 1.7 \\
\hline 3 & 8 & $\left.\begin{array}{l}0 \\
0\end{array}\right\}$ & 0.599 & 13.4 & 16.0 & -2.6 & -1 & $\begin{array}{l}3 \\
4\end{array}$ & $\left.\begin{array}{l}1 \\
1\end{array}\right\}$ & 0.246 & 113.8 & 115.0 & -1.2 \\
\hline & $\begin{array}{r}12 \\
0\end{array}$ & $0)$ & 0.616 & 196 & 238 & & 1 & 2 & 1 & - & - & 4.9 & 4.9 \\
\hline 4 & 1 & 0 & & 19.0 & 23.8 & -4.2 & 1 & 3 & $1\}$ & 0.274 & 29.1 & 26.6 & 2.5 \\
\hline $\begin{array}{l}2 \\
4\end{array}$ & $\begin{array}{r}11 \\
2\end{array}$ & $\left.\begin{array}{l}0 \\
0\end{array}\right)$ & & & & & $\begin{array}{r}-1 \\
0\end{array}$ & $\begin{array}{l}1 \\
5\end{array}$ & $\begin{array}{l}1) \\
1\end{array}$ & 0.286 & 5.7 & 4.0 & 1.7 \\
\hline 4 & 3 & $0\}$ & 0.631 & 25.4 & 15.4 & 4.0 & 1 & 4 & 1 & 0.304 & 9.1 & 8.1 & 1.0 \\
\hline 3 & 9 & 0 & & & & & -1 & 5 & 1 & 0.312 & 17.7 & 16.3 & 1.4 \\
\hline 4 & 4 & 0 & 0.640 & 25.0 & 22.8 & 2.2 & -2 & 1 & $1)$ & 0.324 & 8.5 & 12.6 & -4.1 \\
\hline 1 & 13 & 0 & - & - & 1.8 & 1.8 & 0 & 6 & $1\}$ & & & & \\
\hline $\begin{array}{l}2 \\
4\end{array}$ & $\begin{array}{r}12 \\
5\end{array}$ & $\left.\begin{array}{l}0 \\
0\end{array}\right\}$ & 0.654 & 11.2 & 9.8 & 1.4 & $\begin{array}{r}-2 \\
1\end{array}$ & $\begin{array}{l}2 \\
5\end{array}$ & $\left.\begin{array}{l}1 \\
1\end{array}\right\}$ & 0.335 & 13.9 & 13.0 & 0.9 \\
\hline 3 & 10 & 0 & - & - & 3.4 & 3.4 & -1 & 6 & 13 & 0.351 & 24.9 & 24.4 & 0.5 \\
\hline 4 & 6 & 0 ? & 0.676 & 14.6 & 9.4 & 5.2 & -2 & 3 & $1\}$ & & & & \\
\hline 0 & 14 & 0 & & & & & 2 & 1 & 1 & 0.364 & 13.6 & 13.7 & -0.1 \\
\hline
\end{tabular}


The Order of the Chains in Ipp Crystals

Table Ib. (Continued)

\begin{tabular}{|c|c|c|c|c|c|c|c|c|c|c|c|c|c|}
\hline$h$ & $k$ & $l$ & $d^{-1} \AA$ & $\left|F_{0}\right|$ & $\left|F_{\text {int }}\right|$ & $F_{\mathrm{d}}$ & $h$ & $k$ & $l$ & $d^{-1} \AA$ & $\left|F_{0}\right|$ & $\left|F_{\text {int }}\right|$ & $F_{\mathrm{d}}$ \\
\hline $\begin{array}{r}0 \\
-2 \\
1\end{array}$ & $\begin{array}{l}7 \\
4 \\
6\end{array}$ & $\left.\begin{array}{l}1 \\
1 \\
1\end{array}\right\}$ & 0.373 & 41.7 & 40.1 & 1.6 & $\begin{array}{r}0 \\
-4 \\
4\end{array}$ & $\begin{array}{r}13 \\
5 \\
1\end{array}$ & $\left.\begin{array}{l}1 \\
1 \\
1\end{array}\right\}$ & 0.648 & 3.7 & 9.9 & -6.2 \\
\hline & & $1)$ & & & & & -1 & 13 & $1)$ & 0.656 & 32.5 & 28.2 & 4.3 \\
\hline $\begin{array}{r}2 \\
-1\end{array}$ & $\begin{array}{l}3 \\
7\end{array}$ & $\left.\begin{array}{l}1 \\
1\end{array}\right\}$ & 0.390 & 39.2 & 38.3 & 0.9 & $\begin{array}{r}-2 \\
4\end{array}$ & $\begin{array}{r}12 \\
2\end{array}$ & $\begin{array}{l}1 \\
1\end{array}$ & & & & \\
\hline-2 & 5 & 1 & 0.400 & 14.6 & 16.7 & -2.1 & $\begin{array}{r}3 \\
-3\end{array}$ & $\begin{array}{r}9 \\
10\end{array}$ & $\begin{array}{l}1 \\
1\end{array}$ & & & & \\
\hline $\begin{array}{l}2 \\
1\end{array}$ & $\begin{array}{l}4 \\
7\end{array}$ & $\left.\begin{array}{l}1 \\
1\end{array}\right\}$ & 0.409 & 27.3 & 26.5 & 0.8 & $\begin{array}{l}4 \\
1\end{array}$ & $\begin{array}{r}3 \\
13\end{array}$ & $\begin{array}{l}1 \\
1\end{array}$ & & & & \\
\hline 0 & 8 & 1 & 0.416 & 22.3 & 22.8 & -0.5 & -4 & 6 & $1)$ & & & & \\
\hline $\begin{array}{l}-2 \\
-1\end{array}$ & $\begin{array}{l}6 \\
8\end{array}$ & $1\}$ & 0.430 & 41.3 & 39.9 & 1.4 & $\begin{array}{l}4 \\
2\end{array}$ & $\begin{array}{r}4 \\
12\end{array}$ & $\left.\begin{array}{l}1 \\
1\end{array}\right\}$ & - & - & 3.4 & 3.4 \\
\hline 2 & 5 & $1 \int$ & & & & & -4 & 7 & $1)$ & 0.693 & 7.5 & 9.2 & -1.7 \\
\hline 1 & 8 & 1 & - & - & 4.1 & 4.1 & $\begin{array}{l}0 \\
4\end{array}$ & $\begin{array}{r}14 \\
5\end{array}$ & $\left.\begin{array}{l}1 \\
1\end{array}\right\}$ & & & & \\
\hline 0 & 9 & $1)$ & 0.467 & 29.0 & 29.3 & -0.3 & 3 & 10 & 1 & - & - & 1.0 & 1.0 \\
\hline $\begin{array}{r}-3 \\
2\end{array}$ & $\begin{array}{l}1 \\
6\end{array}$ & $\left.\begin{array}{l}1 \\
1\end{array}\right\}$ & & & & & -3 & 11 & 1 & 0.704 & 15.1 & 14.7 & 0.4 \\
\hline$-\overline{2}$ & 7 & 1 & & & & & 0 & 0 & 2 & 0.310 & 8.9 & 7.4 & 1.5 \\
\hline & 2 & 1) & & & & & 0 & 1 & 2 & 0.312 & 10.9 & 13.0 & -2.1 \\
\hline $\begin{array}{l}-3 \\
-1\end{array}$ & $\begin{array}{l}3 \\
9\end{array}$ & $\left.\begin{array}{l}1 \\
1\end{array}\right\}$ & 0.481 & 37.1 & 34.1 & 3.0 & $\begin{array}{r}-1 \\
0\end{array}$ & $\begin{array}{l}0 \\
2\end{array}$ & $\left.\begin{array}{l}2 \\
2\end{array}\right\}$ & 0.322 & 32.7 & 34.5 & -1.8 \\
\hline 1 & 9 & 1 & 0.498 & 10.2 & 19.7 & -9.5 & -1 & 1 & $2)$ & & & & \\
\hline $\begin{array}{r}2 \\
-3 \\
-2\end{array}$ & $\begin{array}{l}7 \\
4 \\
8\end{array}$ & $\begin{array}{l}1 \\
1 \\
1\end{array}$ & & & & & $\begin{array}{r}-1 \\
0\end{array}$ & $\begin{array}{l}2 \\
3\end{array}$ & $\left.\begin{array}{l}2 \\
2\end{array}\right\}$ & 0.338 & 4.2 & 4.0 & 0.2 \\
\hline $\begin{array}{r}-2 \\
0\end{array}$ & $\begin{array}{r}8 \\
10\end{array}$ & 1 & & & & & -1 & 3 & 2 & 0.356 & 2.0 & 0.3 & 1.7 \\
\hline $\begin{array}{l}0 \\
3\end{array}$ & $\begin{array}{r}10 \\
1\end{array}$ & $\left.\begin{array}{l}1 \\
1\end{array}\right\}$ & 0.506 & 30.5 & 22.8 & 7.7 & $\begin{array}{l}0 \\
1\end{array}$ & $\begin{array}{l}4 \\
0\end{array}$ & $\left.\begin{array}{l}2 \\
2\end{array}\right\}$ & 0.370 & 20.4 & 18.2 & 2.2 \\
\hline $\begin{array}{r}3 \\
-3\end{array}$ & $\begin{array}{l}2 \\
5\end{array}$ & $\left.\begin{array}{l}1 \\
1\end{array}\right\}$ & 0.516 & 29.8 & 36.0 & -6.2 & 1 & 1 & $2 \int$ & & & & \\
\hline $\begin{array}{l}-3 \\
-1\end{array}$ & 10 & $1\}$ & & & & & $\begin{array}{r}-1 \\
1\end{array}$ & $\begin{array}{l}4 \\
2\end{array}$ & $\left.\begin{array}{l}2 \\
2\end{array}\right\}$ & 0.378 & 7.2 & 10.5 & -3.3 \\
\hline $\begin{array}{l}3 \\
2\end{array}$ & $\begin{array}{l}3 \\
8\end{array}$ & $\left.\begin{array}{l}1 \\
1\end{array}\right\}$ & 0.525 & 11.3 & 13.6 & -2.3 & $\begin{array}{l}0 \\
1\end{array}$ & $\begin{array}{l}5 \\
3\end{array}$ & $\left.\begin{array}{l}2 \\
2\end{array}\right\}$ & 0.392 & 10.6 & 10.2 & 0.4 \\
\hline $\begin{array}{r}1 \\
3 \\
-2\end{array}$ & $\begin{array}{r}10 \\
4 \\
9\end{array}$ & $\left.\begin{array}{l}1 \\
1 \\
1\end{array}\right\}$ & 0.538 & 16.0 & 12.3 & 3.7 & $\begin{array}{r}1 \\
-2 \\
-2 \\
-1\end{array}$ & $\begin{array}{l}5 \\
0 \\
1 \\
5\end{array}$ & $\left.\begin{array}{l}2 \\
2 \\
2\end{array}\right\}$ & 0.402 & 10.8 & 17.0 & -6.2 \\
\hline-3 & 6 & 1 & - & - & 0.1 & 0.1 & -2 & 2 & $2\}$ & 0.417 & 4.2 & 4.7 & -0.5 \\
\hline $\begin{array}{l}0 \\
3\end{array}$ & $\begin{array}{r}11 \\
5\end{array}$ & $\left.\begin{array}{l}1 \\
1\end{array}\right\}$ & 0.555 & 19.9 & 19.5 & 0.4 & 1 & 4 & $2\}$ & & & & \\
\hline $\begin{array}{r}2 \\
-1 \\
-3\end{array}$ & $\begin{array}{r}9 \\
11 \\
7\end{array}$ & $\left.\begin{array}{l}1 \\
1 \\
1\end{array}\right\}$ & 0.567 & 15.1 & 11.9 & 3.2 & $\begin{array}{r}-2 \\
0 \\
-1\end{array}$ & $\begin{array}{l}3 \\
6 \\
6\end{array}$ & $\left.\begin{array}{l}2 \\
2 \\
2\end{array}\right\}$ & 0.428 & $\begin{array}{r}4.4 \\
17.1\end{array}$ & $\begin{array}{r}7.6 \\
17.2\end{array}$ & $\begin{array}{l}-3.2 \\
-0.1\end{array}$ \\
\hline $\begin{array}{r}-2 \\
1\end{array}$ & $\begin{array}{l}10 \\
11\end{array}$ & $\left.\begin{array}{l}1 \\
1\end{array}\right\}$ & 0.578 & 27.3 & 27.5 & -0.2 & $\begin{array}{r}1 \\
-2\end{array}$ & $\begin{array}{l}5 \\
4\end{array}$ & $\left.\begin{array}{l}2 \\
2\end{array}\right\}$ & 0.446 & 8.6 & 8.2 & 0.4 \\
\hline 3 & 6 & $1)$ & & & & & 0 & 7 & 2 & 0.458 & 4.0 & 4.8 & -0.8 \\
\hline $\begin{array}{r}0 \\
-3 \\
2 \\
-4 \\
3\end{array}$ & $\begin{array}{r}12 \\
8 \\
10 \\
1 \\
7\end{array}$ & $\left.\begin{array}{l}1 \\
1 \\
1 \\
1 \\
1\end{array}\right\}$ & 0.599 & 22.6 & 22.9 & -0.3 & $\begin{array}{r}-2 \\
-1 \\
1 \\
2 \\
2\end{array}$ & $\begin{array}{l}5 \\
7 \\
6 \\
0 \\
1\end{array}$ & $\left.\begin{array}{l}2 \\
2 \\
2 \\
2 \\
2\end{array}\right\}$ & 0.469 & 26.7 & 29.7 & -3.0 \\
\hline-4 & 2 & $1\}$ & 0.615 & 12.7 & 13.5 & -0.8 & 2 & 2 & 2 & - & - & 1.0 & 1.0 \\
\hline $\begin{array}{r}-1 \\
-2 \\
-4 \\
1\end{array}$ & $\begin{array}{r}12 \\
11 \\
3 \\
12\end{array}$ & $\left.\begin{array}{l}1 \\
1 \\
1 \\
1\end{array}\right\}$ & 0.623 & 6.2 & 10.9 & -4.7 & $\begin{array}{r}2 \\
-2 \\
0 \\
1\end{array}$ & $\begin{array}{l}3 \\
6 \\
8 \\
7\end{array}$ & $\left.\begin{array}{l}2 \\
2 \\
2 \\
2\end{array}\right\}$ & 0.498 & 17.3 & 21.5 & -4.2 \\
\hline $\begin{array}{l}-3 \\
-4\end{array}$ & $\begin{array}{l}9 \\
4\end{array}$ & $1\}$ & 0.635 & 14.9 & 14.3 & 0.6 & $\begin{array}{r}-1 \\
2\end{array}$ & $\begin{array}{l}8 \\
4\end{array}$ & $\left.\begin{array}{l}2 \\
2\end{array}\right\}$ & 0.507 & 3.7 & 2.8 & 0.9 \\
\hline $\begin{array}{l}3 \\
2\end{array}$ & $\begin{array}{r}8 \\
11\end{array}$ & $\begin{array}{l}1) \\
1\end{array}$ & - & - & 3.1 & 3.1 & $\begin{array}{l}-3 \\
-3\end{array}$ & $\begin{array}{l}0 \\
1\end{array}$ & $\left.\begin{array}{l}2 \\
2\end{array}\right\}$ & 0.518 & 14.4 & 12.0 & 2.4 \\
\hline
\end{tabular}


M. Hikosaka and T. Seto

Table Ib. (Continued)

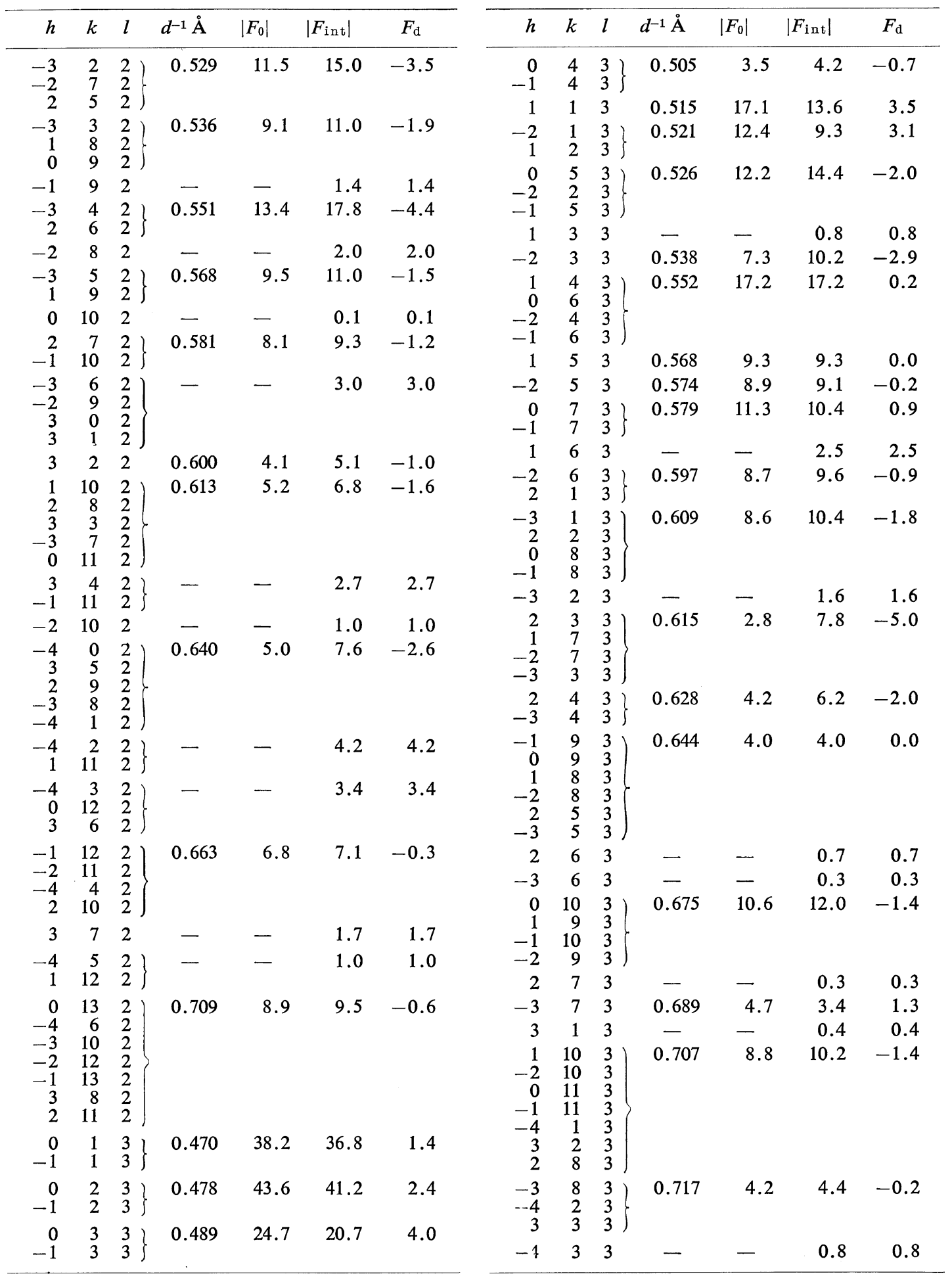


The Order of the Chains in Ipp Crystals

Table Ib. (Continued)

\begin{tabular}{|c|c|c|c|c|c|c|c|c|c|c|c|c|c|}
\hline$h$ & $k$ & $l$ & $d^{-1} \AA$ & $\left|F_{0}\right|$ & $\left|F_{\text {int }}\right|$ & $F_{\mathrm{d}}$ & $h$ & $k$ & $l$ & $d^{-1} \AA$ & $\left|F_{0}\right|$ & $\left|F_{\text {int }}\right|$ & $F_{\mathrm{d}}$ \\
\hline 3 & 4 & 3 & 0.729 & 4.1 & 1.7 & 2.4 & 0 & 7 & $4)$ & 0.713 & 16.7 & 19.6 & -2.9 \\
\hline-4 & 4 & 3 & - & - & 1.3 & 1.3 & $\begin{array}{l}-2 \\
-3\end{array}$ & $\begin{array}{l}6 \\
0\end{array}$ & $\left.\begin{array}{l}4 \\
4\end{array}\right\}$ & & & & \\
\hline 2 & 9 & 3 & - & - & 0.4 & 0.4 & -3 & 1 & 4 & & & & \\
\hline 1 & 11 & 3 & - & - & 2.5 & 2.5 & -3 & 2 & 4 & - & - & 2.3 & 2.3 \\
\hline-2 & 11 & 3 & 0.743 & 7.4 & 9.3 & -1.9 & 1 & 6 & 4 & - & - & 0.6 & 0.6 \\
\hline $\begin{array}{r}-3 \\
3 \\
0 \\
-1 \\
-4\end{array}$ & $\begin{array}{r}9 \\
5 \\
12 \\
12 \\
5\end{array}$ & $\left.\begin{array}{l}3 \\
3 \\
3 \\
3 \\
3\end{array}\right\}$ & & & & & $\begin{array}{r}-1 \\
-2 \\
-3 \\
0 \\
2\end{array}$ & $\begin{array}{l}8 \\
7 \\
3 \\
8 \\
0\end{array}$ & $\left.\begin{array}{l}4 \\
4 \\
4 \\
4 \\
4\end{array}\right\}$ & 0.734 & 17.8 & 18.4 & -0.6 \\
\hline 3 & 6 & 3 & - & - & 4.0 & 4.0 & $\begin{array}{l}2 \\
?\end{array}$ & 1 & $4)$ & & & & \\
\hline $\begin{array}{r}-4 \\
2 \\
-3\end{array}$ & $\begin{array}{r}6 \\
10 \\
10\end{array}$ & $\left.\begin{array}{l}3 \\
3 \\
3\end{array}\right\}$ & 0.766 & 6.8 & 7.5 & -0.7 & $\begin{array}{r}2 \\
-3 \\
1 \\
2\end{array}$ & $\begin{array}{l}2 \\
4 \\
7 \\
3\end{array}$ & $\left.\begin{array}{l}4 \\
4 \\
4 \\
4\end{array}\right\}$ & 0.746 & 4.5 & 8.8 & -4.3 \\
\hline 1 & 12 & 3 & - & - & 2.4 & 2.4 & -3 & 5 & $4)$ & 0.757 & 29.0 & 29.2 & -0.2 \\
\hline-2 & 12 & 3 & - & - & 3.0 & 3.0 & $\begin{array}{r}0 \\
-1\end{array}$ & $\begin{array}{l}9 \\
9\end{array}$ & $\left.\begin{array}{l}4 \\
4\end{array}\right\}$ & & & & \\
\hline 3 & 7 & 3 & - & - & 3.5 & 3.5 & -1 & 4 & 4 & & & & \\
\hline 0 & 13 & $3)$ & 0.783 & 6.4 & 6.5 & -0.1 & -2 & 8 & $4)$ & & & & \\
\hline $\begin{array}{l}-1 \\
-4\end{array}$ & $\begin{array}{r}13 \\
7\end{array}$ & $\left.\begin{array}{l}3 \\
3\end{array}\right\}$ & & & & & $\begin{array}{r}1 \\
-3\end{array}$ & $\begin{array}{l}8 \\
6\end{array}$ & $\left.\begin{array}{l}4 \\
4\end{array}\right\}$ & 0.770 & 7.2 & 9.2 & -2.0 \\
\hline $\begin{array}{r}2 \\
-3 \\
3\end{array}$ & $\begin{array}{r}11 \\
11 \\
8\end{array}$ & $\left.\begin{array}{l}3 \\
3 \\
3\end{array}\right\}$ & 0.801 & 6.1 & 6.2 & -0.1 & $\begin{array}{r}-1 \\
-2 \\
0\end{array}$ & $\begin{array}{r}10 \\
9 \\
10 \\
7\end{array}$ & $\left.\begin{array}{l}4 \\
4 \\
4\end{array}\right\}$ & 0.785 & 5.2 & 7.5 & -2.3 \\
\hline $\begin{array}{l}-1 \\
-1\end{array}$ & $\begin{array}{l}0 \\
1\end{array}$ & $\left.\begin{array}{l}4 \\
4\end{array}\right\}$ & 0.620 & 8.9 & 9.2 & -0.3 & $\begin{array}{r}-3 \\
2\end{array}$ & $\begin{array}{l}7 \\
6\end{array}$ & $\left.\begin{array}{l}4 \\
4\end{array}\right)$ & & & & \\
\hline $\begin{array}{r}0 \\
0 \\
-1 \\
0\end{array}$ & $\begin{array}{l}0 \\
1 \\
2 \\
2\end{array}$ & $\left.\begin{array}{l}4 \\
4 \\
4 \\
4\end{array}\right\}$ & 0.624 & 10.9 & 10.2 & 0.7 & $\begin{array}{r}1 \\
-4 \\
-4 \\
-2 \\
-4\end{array}$ & $\begin{array}{r}9 \\
0 \\
1 \\
10 \\
2\end{array}$ & $\left.\begin{array}{l}4 \\
4 \\
4 \\
4 \\
4\end{array}\right\}$ & $\overline{0 .}$ & $\overline{14.4}$ & $\begin{array}{r}1.0 \\
12.8\end{array}$ & $\begin{array}{l}1.0 \\
1.6\end{array}$ \\
\hline 0 & 2 & 4 & 0.630 & 6.8 & 6.8 & 0.0 & 2 & 7 & $4)$ & & & & \\
\hline-1 & 3 & 4 & 0.634 & 4.1 & 4.1 & 0.0 & -1 & 11 & $4)$ & 0.818 & 7.0 & 7.6 & 0.6 \\
\hline 0 & 3 & 4 & 0.639 & 5.1 & 3.1 & 2.0 & $\begin{array}{l}-3 \\
-4\end{array}$ & $\begin{array}{l}8 \\
3\end{array}$ & $\left.\begin{array}{l}4 \\
4\end{array}\right\}$ & & & & \\
\hline $\begin{array}{l}-1 \\
-2 \\
-2\end{array}$ & $\begin{array}{l}4 \\
0 \\
1\end{array}$ & $\left.\begin{array}{l}4 \\
4 \\
4\end{array}\right\}$ & 0.651 & 10.6 & 11.0 & -0.4 & $\begin{array}{r}-4 \\
0 \\
1\end{array}$ & $\begin{array}{l}3 \\
11 \\
10\end{array}$ & $\left.\begin{array}{l}4 \\
4 \\
4\end{array}\right\}$ & & & & \\
\hline $\begin{array}{r}-2 \\
0\end{array}$ & $\begin{array}{l}1 \\
4\end{array}$ & $\left.\begin{array}{l}4 \\
4\end{array}\right)$ & & & & & $\begin{array}{r}-4 \\
3\end{array}$ & $\begin{array}{l}4 \\
0\end{array}$ & $\left.\begin{array}{l}4 \\
4\end{array}\right)$ & 0.826 & 12.6 & 10.2 & 2.4 \\
\hline-2 & 2 & 4 & 0.658 & 7.5 & 8.2 & -0.7 & 3 & 1 & $4\}$ & & & & \\
\hline 1 & 0 & $4)$ & 0.665 & 26.1 & 21.5 & 4.6 & 2 & 8 & 4) & & & & \\
\hline $\begin{array}{r}-1 \\
1\end{array}$ & $\begin{array}{l}5 \\
1\end{array}$ & $\begin{array}{l}4 \\
4\end{array}$ & & & & & 3 & 2 & 4 & - & - & 3.5 & 3.5 \\
\hline $\begin{array}{r}1 \\
-2 \\
0 \\
1\end{array}$ & $\begin{array}{l}1 \\
3 \\
5 \\
2\end{array}$ & $\left.\begin{array}{l}4 \\
4 \\
4 \\
4\end{array}\right\}$ & & & & & $\begin{array}{r}-3 \\
-4 \\
-2 \\
3\end{array}$ & $\begin{array}{r}9 \\
5 \\
11 \\
3\end{array}$ & $\left.\begin{array}{l}4 \\
4 \\
4 \\
4\end{array}\right\}$ & 0.835 & 15.8 & 15.3 & 0.5 \\
\hline $\begin{array}{r}-2 \\
1 \\
-1\end{array}$ & $\begin{array}{l}4 \\
3 \\
6\end{array}$ & $\left.\begin{array}{l}4 \\
4 \\
4\end{array}\right\}$ & 0.680 & 21.8 & 19.4 & 2.4 & $\begin{array}{r}-1 \\
3 \\
-4\end{array}$ & $\begin{array}{r}12 \\
4 \\
6\end{array}$ & $\left.\begin{array}{l}4 \\
4 \\
4\end{array}\right)$ & 0.849 & 9.9 & 9.8 & 0.1 \\
\hline $\begin{array}{l}0 \\
1\end{array}$ & $\begin{array}{l}6 \\
4\end{array}$ & $\left.\begin{array}{l}4 \\
4\end{array}\right\}$ & 0.688 & 8.6 & 12.8 & -4.2 & $\begin{array}{r}-4 \\
0 \\
1\end{array}$ & $\begin{array}{l}0 \\
12 \\
11\end{array}$ & $\left.\begin{array}{l}4 \\
4 \\
4\end{array}\right\}$ & & & & \\
\hline-2 & 5 & 4 & - & - & 1.7 & 1.7 & 2 & 9 & $4)$ & & & & \\
\hline $\begin{array}{r}-1 \\
1\end{array}$ & $\begin{array}{l}7 \\
5\end{array}$ & $\left.\begin{array}{l}4 \\
4\end{array}\right\}$ & 0.706 & 6.5 & 5.1 & 1.4 & $\begin{array}{r}-3 \\
3\end{array}$ & $\begin{array}{r}10 \\
5\end{array}$ & $\left.\begin{array}{l}4 \\
4\end{array}\right\}$ & 0.860 & 9.2 & 5.9 & 3.3 \\
\hline
\end{tabular}

$\sum\left|F_{\text {int }}\right|=2963.0, \quad \sum\left|F_{\mathrm{d}}\right|=449.9, \quad R=0.152$.

a Observed on the specimen drawn $700 \%$ and annealed under isometric conditions at $171^{\circ} \mathrm{C}$ for $2 \mathrm{hr}$.

b $\left|F_{\text {int }}\right|=\left|0.34 F_{\mathrm{C} 2 / \mathrm{c}}+0.66 F_{\mathrm{P}_{1} / \mathrm{c}}\right|$.

c $F_{\mathrm{d}}=\left|F_{0}\right|-\left|F_{\text {int }}\right|$. 


\section{Hikosaka and T. Seto}

Table II. Comparison between the observed and calculated structure factors of the ipp $\alpha 1$ form. $F_{0}$ and $F_{\mathrm{c}}$ are obtained as in Table Ib, exept that the values of $M$ are taken as follows: $M=\frac{1}{2}$ for $h 0 l, M=1$ for $h k 0$, and $M=1 / \sqrt{2}$ for the others.

\begin{tabular}{|c|c|c|c|c|c|c|c|c|c|c|c|}
\hline$h$ & $k$ & $l$ & $\left|F_{0}\right|^{\mathrm{a}}$ & $\left|F_{\mathrm{c}}\right|^{\mathrm{b}}$ & $F_{\mathrm{d}} \mathrm{c}$ & $h$ & $k$ & $l$ & $\left|F_{0}\right|$ & $\left|F_{\mathrm{c}}\right|$ & $F_{\mathrm{d}}$ \\
\hline$l=0$ & & & & & & 3 & 15 & 0 & - & 3.1 & -3.1 \\
\hline 0 & 2 & 0 & - & 0.6 & -0.6 & 0 & 18 & 0 ? & 5.9 & 4.7 & 1.2 \\
\hline 1 & 1 & 0 & 67.5 & 64.0 & 3.5 & 5 & 9 & $0\}$ & & & \\
\hline 0 & 4 & 0 & 55.2 & 59.0 & -3.8 & $l=1$ & & & & & \\
\hline 1 & 3 & 0 & 60.4 & 70.1 & -9.7 & -1 & 1 & 1 & 4.2 & 5.0 & -0.8 \\
\hline $\begin{array}{l}1 \\
0\end{array}$ & $\begin{array}{l}5 \\
6\end{array}$ & $\left.\begin{array}{l}0 \\
0\end{array}\right\}$ & 34.6 & 35.9 & -1.3 & 1 & 1 & 1 & 57.1 & 54.1 & 3.0 \\
\hline 2 & 0 & 0 & 19.5 & 23.5 & -4.0 & $\begin{array}{r}-1 \\
0\end{array}$ & $\begin{array}{l}3 \\
4\end{array}$ & $\left.\begin{array}{l}1 \\
1\end{array}\right\}$ & 72.7 & 81.3 & -8.6 \\
\hline 2 & 2 & 0 & 32.2 & 35.2 & -3.0 & 1 & 3 & 1 & 20.2 & 17.9 & 2.3 \\
\hline $\begin{array}{l}2 \\
1\end{array}$ & $\begin{array}{l}4 \\
7\end{array}$ & $\left.\begin{array}{l}0 \\
0\end{array}\right\}$ & 15.7 & 16.0 & -0.3 & -1 & 5 & 1 & 11.4 & 11.5 & -0.1 \\
\hline 0 & 8 & 0 & 3.1 & 8.4 & -5.3 & 0 & 6 & $1)$ & 6.7 & 12.2 & -5.7 \\
\hline 2 & 6 & 0 & 12.0 & 16.8 & -4.8 & -2 & 2 & $1\}$ & & & \\
\hline 3 & 1 & $0\}$ & 20.8 & 19.3 & 1.5 & 1 & 5 & $1)$ & & & \\
\hline 1 & 9 & $0\}$ & & & & -2 & 4 & $1\}$ & 23.2 & 24.4 & -1.2 \\
\hline 0 & 10 & $0\}$ & - & 7.9 & -7.9 & 2 & 2 & $1\}$ & & & \\
\hline 3 & 3 & $0\}$ & & & & -1 & 7 & 1 & 20.0 & 20.8 & -0.8 \\
\hline 2 & 8 & 0 & 12.0 & 14.7 & -2.7 & 2 & 4 & $1\}$ & 28.8 & 24.6 & 4.2 \\
\hline 3 & 5 & 0 & 4.5 & 7.7 & -3.2 & 0 & 8 & $1\}$ & & & \\
\hline 1 & 11 & 0 & - & 3.0 & -3.0 & -2 & 6 & 1 & 25.6 & 25.6 & -1.2 \\
\hline $\begin{array}{l}3 \\
2\end{array}$ & $\begin{array}{r}7 \\
10\end{array}$ & $\left.\begin{array}{l}0 \\
0\end{array}\right\}$ & 7.5 & 10.3 & -2.8 & $\begin{array}{r}-3 \\
2\end{array}$ & $\begin{array}{l}1 \\
6\end{array}$ & $\left.\begin{array}{l}1 \\
1\end{array}\right\}$ & 10.8 & 9.0 & 1.8 \\
\hline 0 & 12 & 0 & 7.5 & 10.6 & -3.1 & -3 & 3 & $1\}$ & 22.9 & 24.1 & -1.2 \\
\hline 4 & 0 & $0\}$ & 4.0 & 6.9 & -2.9 & -1 & 9 & $1\}$ & & & \\
\hline $\begin{array}{l}4 \\
3\end{array}$ & $\begin{array}{l}2 \\
9\end{array}$ & $\begin{array}{l}0\} \\
0\end{array}$ & & & & 1 & 9 & 1 & - & 1.1 & -1.1 \\
\hline $\begin{array}{l}3 \\
4\end{array}$ & $\begin{array}{l}9 \\
4\end{array}$ & $\left.\begin{array}{l}0 \\
0\end{array}\right\}$ & 16.3 & 16.1 & 0.2 & -2 & 8 & $1)$ & 20.6 & 16.6 & 4.0 \\
\hline 1 & 13 & 0 & - & 0.9 & -0.9 & $\begin{array}{l}0 \\
3\end{array}$ & $\begin{array}{r}10 \\
1\end{array}$ & $\left.\begin{array}{l}1 \\
1\end{array}\right\}$ & & & \\
\hline 2 & 12 & 0 & - & 4.1 & -4.1 & -3 & 5 & $1\}$ & 13.7 & 15.7 & -2.0 \\
\hline 4 & 6 & $0\}$ & 6.4 & 4.7 & 1.7 & $\begin{array}{r}-3 \\
3\end{array}$ & 3 & $1\}$ & & & \\
\hline 0 & 14 & $0\}$ & & & & 2 & 8 & 1 & - & 4.2 & -4.2 \\
\hline 3 & 11 & 0 & - & 2.4 & -2.4 & 3 & 5 & $1)$ & 11.3 & 13.0 & -1.7 \\
\hline 4 & 8 & 0 & 7.8 & 8.4 & -0.6 & $\begin{array}{r}3 \\
-1\end{array}$ & 11 & $1\}$ & & & \\
\hline 2 & 14 & $0\}$ & 14.1 & 16.5 & -2.4 & -3 & 7 & 1) & & & \\
\hline 1 & 15 & $0\}$ & & & & -2 & 10 & $1\}$ & 20.6 & 17.8 & 2.8 \\
\hline 5 & 1 & $0\}$ & 11.3 & 10.0 & 1.3 & $\overline{1}$ & 11 & $1\}$ & & & \\
\hline 0 & 16 & $0\}$ & & & & 0 & 12 & $1)$ & 14.4 & 14.0 & 0.4 \\
\hline $\begin{array}{l}5 \\
4 \\
3\end{array}$ & $\begin{array}{r}3 \\
10 \\
13\end{array}$ & $\left.\begin{array}{l}0 \\
0 \\
0\end{array}\right\}$ & 9.1 & 13.6 & -4.5 & $\begin{array}{r}2 \\
3 \\
-4\end{array}$ & $\begin{array}{r}10 \\
7 \\
2\end{array}$ & $\left.\begin{array}{l}1 \\
1 \\
1\end{array}\right\}$ & & & \\
\hline 5 & 5 & 0 & 11.5 & 11.8 & -0.3 & -3 & 9 & 1 & - & 1.6 & -1.6 \\
\hline 2 & 16 & 0 & - & 5.1 & -5.1 & -4 & 4 & 1 & 6.1 & 8.7 & -2.6 \\
\hline $\begin{array}{l}5 \\
1 \\
4\end{array}$ & $\begin{array}{r}7 \\
17 \\
12\end{array}$ & $\left.\begin{array}{l}0 \\
0 \\
0\end{array}\right\}$ & 7.2 & 9.3 & -2.1 & $\begin{array}{r}-1 \\
-2 \\
4\end{array}$ & $\begin{array}{r}13 \\
12 \\
2\end{array}$ & $\left.\begin{array}{l}1 \\
1 \\
1\end{array}\right\}$ & 19.6 & 17.0 & 2.6 \\
\hline
\end{tabular}

$\sum\left|F_{\mathrm{c}}\right|=940.8, \quad \sum\left|F_{\mathrm{d}}\right|=147.2, \quad R=0.157$.

a Observed on the specimen drawn to $700 \%$ and annealed under isometric conditions at $140^{\circ} \mathrm{C}$ for $2 \mathrm{hr}$.

b $F_{\mathrm{c}}=F_{\mathrm{C} 2 / \mathrm{c}}$ and the coordinates given in Table Ia were used.

c $F_{\mathrm{d}}=\left|F_{0}\right|-\left|F_{\mathrm{c}}\right|$. 


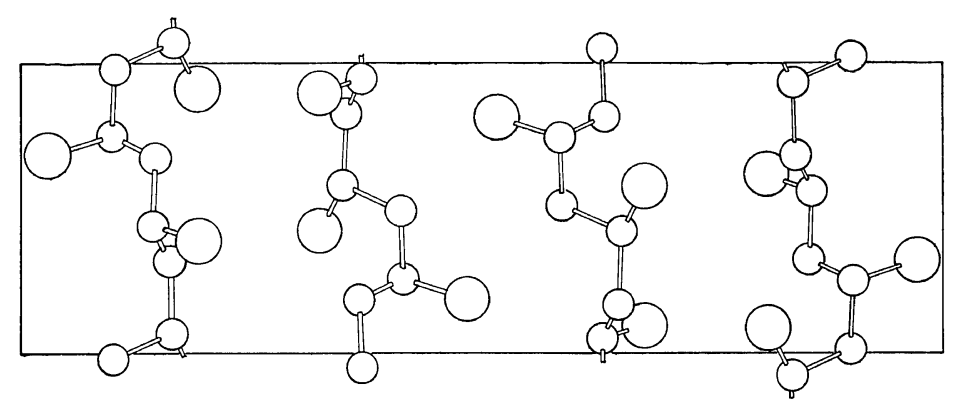

(a)

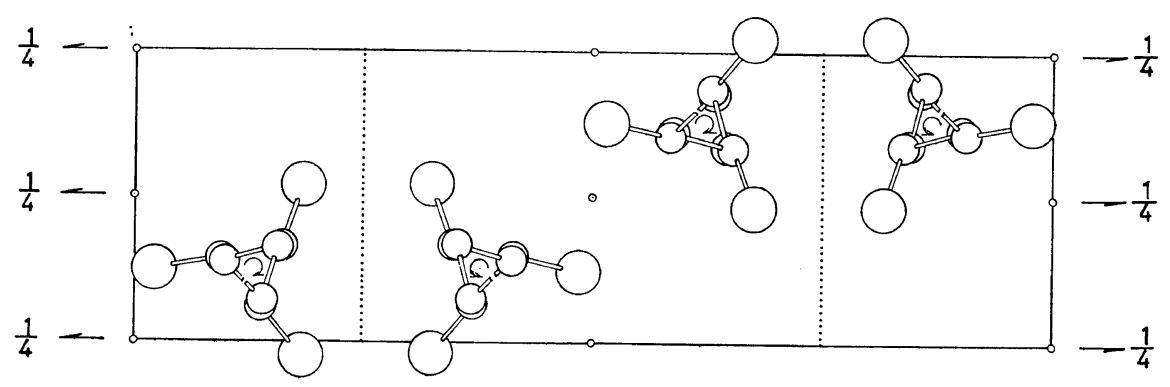

(b)

Figure 9. The unit cell of the $\alpha 2$ form, $P 2_{1} / c$, projected onto (a) (100), (b) (001). Large and small circles indicate methyl-groups and carbon atoms.

cell of the $\alpha 2$ form is illustrated in Figure $9 \mathrm{a}$ and $b$.

\section{Structure of the $\alpha 1$ Form}

The absolute values of the observed structure factor of the $\alpha 1$ form (i.e., of A specimen) generally agree with those given by $\mathrm{Natta}^{1}$; thus it is reasonable to take the same space group $\mathrm{C} 2 / \mathrm{c}$ for the $\alpha 1$ form as given by Natta. The structure factor of the $\alpha 1$ form is calculated from the same atomic coordinates as those of the intermediate form. The results are listed in Table II. The $R$-factor equals 0.157 , which is good enough when the diffuseness of the X-ray pattern is taken into consideration. Therefore we determine the space group of the $\alpha 1$ form to be $\mathrm{C} 2 / \mathrm{c}$.

\section{ORDER DOMAIN EDUCTION MODEL}

Relation Between the Two Structures $\alpha 1$ and $\alpha 2$

The type of ipp chain in the crystal is characterized by two factors: the winding of helices (right or left handed) and the projected direction of the $\mathrm{CH}-\mathrm{CH}_{3}$ bonds onto the $c$-axis (up or down). Hence, there can be two kinds of regularities, corresponding to the two factors of "helical arrangement" and "up or down arrangement" respectively. The characteristics of the chain arrangement in the $\alpha 1$ and $\alpha 2$ forms are schematically illustrated in Figures $10 a$ and $b$. The "helical arrangement" in the $\alpha 1$ form is just the same as that in the $\alpha 2$ form: on every (040) plane, the winding of all the helical chains is the same, and on planes adjacent to each other it is opposite. Such a regular state is named an "order state" as to "helical arrangement." The "up or down arrangement" in the $\alpha 1$ form is, on the other hand, different from that in the $\alpha 2$ form. In the $\alpha 2$ form crystal, the projected direction of the $\mathrm{CH}-\mathrm{CH}_{3}$ bonds onto the $c$-axis is the same on every (200) plane and on adjacent planes it is opposite in direction. In the $\alpha 1$ form crystal, there is no regularity in "up or down arrangement" on every (200) plane. Such states in the $\alpha 2$ and $\alpha 1$ forms will be called "order state" and "disorder state" as to "up or down arrangement."

\section{Conditions for Structural Model}

The structural model for the intermediate 


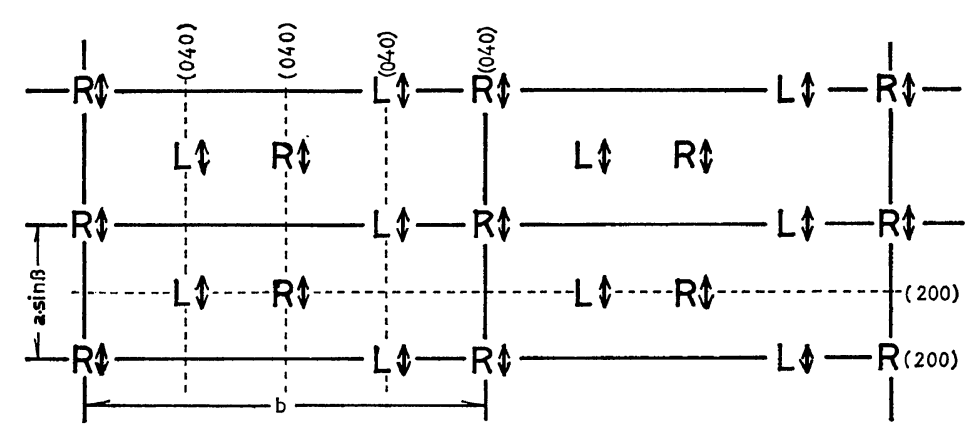

(a) $\mathrm{C} 2 / \mathrm{c}$

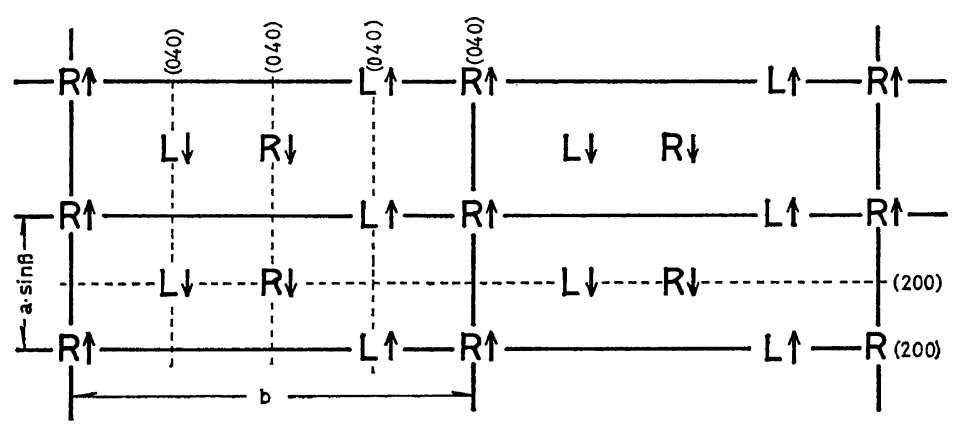

(b) $P 2_{1} / c$

$$
\left\{\begin{array}{l}
\text { Helix; R: right handed, } L \text { : left handed } \\
\mathrm{CH}-\mathrm{CH}_{3} ; \uparrow: \text { up , } \downarrow \text { : down, } \\
\uparrow: \text { up \& down are equally weighted }
\end{array}\right.
$$

Figure 10. Schematic structures of (a) $\alpha 1$ form and (b) $\alpha 2$ form.

form should be able to explain the following experimental results:

(A) if $W$ denotes the line width, it should satisfy the relation $W_{(h+k=\text { odd })}>W_{(h+k=\text { even })}$,

(B) the structure factor $F_{\text {int }}$ of the model should satisfy

$$
\left\{\begin{array}{cl}
\left|F_{\text {int }}\right|<\left|F_{\mathrm{P}_{1} / \mathrm{c}}\right| & \text { (for } h+k=\text { odd }) \\
F_{\text {int }}=F_{\mathrm{P}_{2} / \mathrm{c}} & \text { (for } h+k=\text { even })
\end{array}\right.
$$

In addition, the model should, of course, be able to explain the temperature dependence of the transition mechanism.

We assume hereafter that the sizes of crystallites are large enough to give Bragg reflections and have no coherence with each other. We also assume that each crystallite has an orderly arrangement of helices.

It will be convenient to derive the following relations from eq 5 and 7 :

$$
\begin{cases}F_{\mathrm{C} 2 / \mathrm{c}}=F_{\mathrm{P}_{1} / \mathrm{c}} \neq 0 & \text { for } h+k=\text { even } \\ F_{\mathrm{C} 2 / \mathrm{c}}=0, F_{\mathrm{P}_{1} / \mathrm{c}} \neq 0 & \text { for } h+k=\text { odd }\end{cases}
$$

\section{Order Domain Eduction Model}

We now propose a model for the structure of the intermediate form: within a crystallite, local"order" domains, where the "up or down arrangement" is in "order," are scattered in a "sea" which is in "disorder" like floating islands. The $\alpha 1-\alpha 2$ transition can be explained by eduction and growth of the "order" domains. The $\alpha 1$ and $\alpha 2$ states correspond to states where a crystallite is covered by the "disorder" sea or by the "order" domains. The $F_{\text {int }}$ of this model is given by

$$
\left|F_{\text {int }}\right|=\sqrt{\sum_{i} v_{i}\left|F_{\text {cryst }}^{(i)}\right|^{2}}
$$

where $v_{i}$ and $F_{\text {cryst }}^{(i)}$ are the volume fraction and structure factor of the $i$-th crystallite in a crystal 
respectively and the summation is carried out over the crystal. If \langle\rangle$_{\text {av }}$ denotes the space average over the crystal, we replace eq 10 by the average,

$$
F_{\text {int }} \cong\left\langle F_{\text {cryst }}^{(i)}\right\rangle_{\text {av }}
$$

All waves scattered from molecules within a crystallite are coherent, and therefore, $F_{\text {cryst }}$ can be written in a linear combination

$$
F_{\mathrm{cryst}}^{(i)}=r F_{\mathrm{C} 2 / \mathrm{c}}+s F_{\mathrm{P}_{1} / \mathrm{c}}(\uparrow)+t F_{\mathrm{P} 2_{1} / \mathrm{c}}(\downarrow)
$$

where $r, s$, and $t$ are volume fractions of $\mathrm{C} 2 / \mathrm{c}$, $\mathbf{P} 2_{1} / \mathrm{c}(\uparrow)$, and $\mathbf{P} 2_{1} / \mathrm{c}(\downarrow)$ regions in the $i$-th crystallite, satisfying

$$
r+s+t=1 \quad \text { and } \quad s>t
$$

Here, $F_{\mathrm{P2}_{1} / \mathrm{c}}(\uparrow)$ and $F_{\mathrm{P2}_{1} / \mathrm{c}}(\downarrow)$ are the structure factors of order domains in which the "up or down arrangements" are quite opposite to each other.

As we show in an Appendix,

$$
F_{\mathrm{P} 2_{1} / \mathrm{c}} / \mathrm{c}(\uparrow)+F_{\mathrm{P2}_{1} / \mathrm{c}}(\downarrow)=2 F_{\mathrm{C} 2 / \mathrm{c}}
$$

eq 12 becomes

$$
\begin{aligned}
F_{\text {cryst }}^{(i)} & =(r+2 t) F_{\mathrm{C} 2 / \mathrm{c}}+(s-t) F_{\mathrm{P}_{1} / \mathrm{c}} \\
& =(2-r-2 s) F_{\mathrm{C} 2 / \mathrm{c}}+(r+2 s-1) F_{\mathrm{P}_{2} / \mathrm{c}}
\end{aligned}
$$

If we put

$$
\langle 2-(r+2 s)\rangle_{\mathrm{av}}=\bar{n}
$$

we have the final formula the same as eq 8:

$$
F_{\text {int }} \cong\left\langle F_{\text {cryst }}^{(i)}\right\rangle_{\mathrm{av}}=\bar{n} F_{\mathrm{C} 2 / \mathrm{c}}+(1-\tilde{n}) F_{\mathrm{P}_{1} / \mathrm{c}}
$$

Thus, we can strictly prove eq 8 . It is clear that eq 8 satisfies the condition $B$, because eq 8 can be rewritten as

$$
\left|F_{\mathrm{int}}\right|\left\{\begin{array}{l}
=(1-\bar{n})\left|F_{\mathrm{P}_{1} / \mathrm{c}}\right|<\left|F_{\mathrm{P}_{1} / \mathrm{c}}\right| \text { for } h+k=\text { odd } \\
=\left|F_{\mathrm{P}_{1} / \mathrm{c}}\right| \text { for } h+k=\text { even }
\end{array}\right.
$$

The condition $\mathrm{A}$ is also satisfied by the model, because $W_{h+l=e v e n}$ is in inverse proportion to the size of a crystallite; while $W_{h+k=\text { odd }}$ is, on the other hand, in inverse proportion to the size of an order domain in a crystallite.

The changes in structure, structure factors, and line width with annealing temperature are indicated schematically in Figure 11.

The process by which order domains increase

Form: $\quad \alpha 1$

intermediate $\quad \alpha 2$

a) Grystallite \& Domain

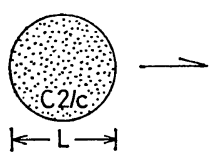

b) Line width
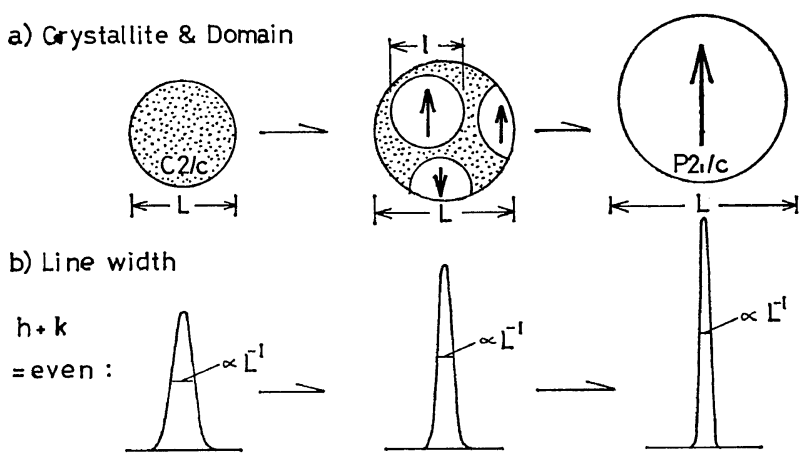

$h+k$

=odd : extinct $\longrightarrow$

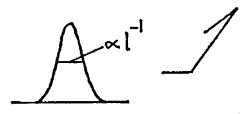

c) Structure factor: F

$$
\left\{\begin{array}{c}
h+k \\
=\text { even } \\
h+k \\
=\text { odd }
\end{array}:\left\{\begin{array}{l}
=F_{c 2 / c} \\
=0
\end{array}\right.\right.
$$

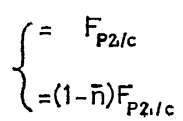

$$
\left\{\begin{array}{l}
=F_{P 2, k} \\
=F_{P 2,1 c}
\end{array}\right.
$$

Figure 11. Scheme of the "order domain eduction model," showing changes in the structure, the line width, and the formula of structure factor. $L$ and 1 denote the size of the crystallite and of the domain. 
in a crystallite can be quantitatively described in terms of a volume fraction $\bar{n}$. As $\left|F_{\text {int }}\right|$ is taken so as to minimize the $R$-factor, we have from eq 17

$$
\left|F_{0}\right| \cong\left|F_{\text {int }}\right|=(1-\bar{n})\left|F_{\mathrm{P}_{1} / \mathrm{c}}\right| \text { for } h+k=\text { odd }
$$

and so

$$
(1-\bar{n}) \cong \frac{\left|F_{0}\right|}{\left|F_{\mathrm{P}_{2} / \mathrm{c}}\right|} \propto I_{0}(T) \text { for } h+k=\text { odd }
$$

Using eq 18 and Figure 2, we have a temperature dependence of $(1-\bar{n})$ which is illustrated in Figure 12. The $(1-\bar{n})$ of the state indicated

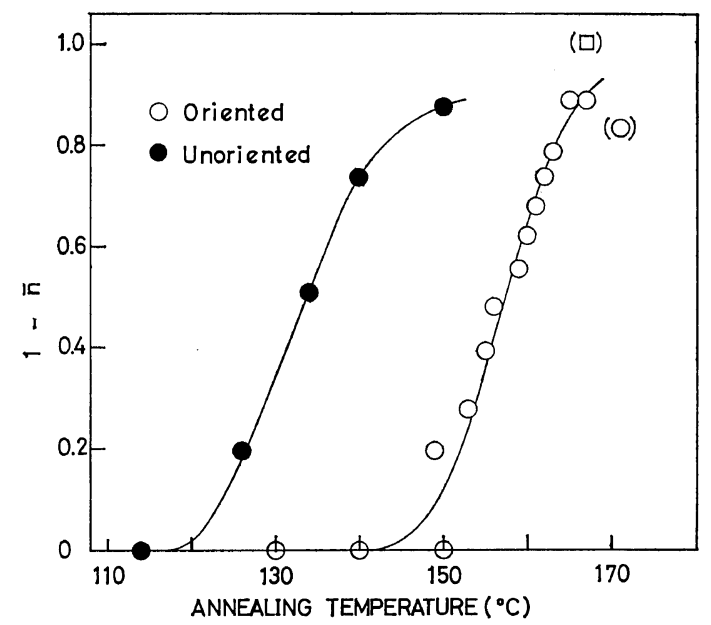

Figure 12. Change in the volume fraction of the $\alpha 2$ form $(1-\bar{n})$ with annealing temperature.

by ( $\square$ ) in Figure 2 can be regarded as very close to unity.

\section{DISCUSSION}

\section{Comparison with Other Structural Analyses}

Natta proposed a model for the $\alpha$ form, in which the crystal was composed of small blocks, each of which corresponded to a $\mathrm{Cc}$ or $\mathrm{C} 2 \% \mathrm{c}$ space group. ${ }^{1}$ Furthermore, he thought, from the fact that the two sets of structure factors, measured and calculated on a $\mathrm{C} 2 / \mathrm{c}$ space group, were in close agreement as the block became small, that the space groups $\mathrm{Cc}$ and $\mathrm{C} 2 / \mathrm{c}$ corresponded to the "order state" and "disorder state" respectively. But his set of two space groups turned out not to predict the appearence of the reflections $h k l$ with $h+k=$ odd. Natta's disorder model $(\mathrm{C} 2 / \mathrm{c})$ is, on the other hand, proved right for the $\alpha 1$ form model, though different values of chain shape and chain packing parameters are taken in our structural analysis.

Mencik proposed a $\mathbf{P 2} / \mathrm{c}$ space group to explain reflections $h k l$ with $h+k=$ odd, $\frac{\overline{2} 31}{161}$, but it was insufficient because chain shape and chain packing were not given, and the structure factors

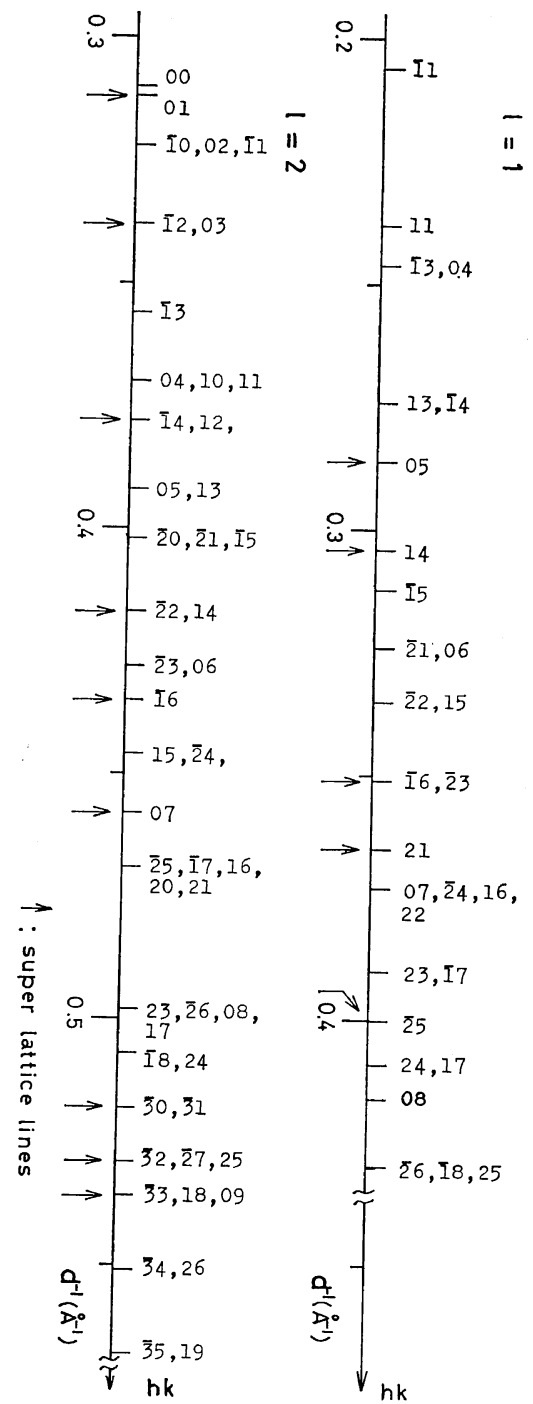

Figure 13. The schematic diagram of the X-ray pattern of the $\alpha 2$ form, where 1 and 11,13 , etc., denote layer line number and $h k$, respectively. 
calculated were not compared with those observed. ${ }^{2}$

We have observed the $\alpha 1-\alpha 2$ transition $^{4}$ and reported preliminarily on these structures, ${ }^{5}$ which are now shown in detail in this paper.

\section{Nature of the $\alpha 1-\alpha 2$ Transition}

The experimental result that the $\alpha 2$ form does not return to the $\alpha 1$ form when it is cooled down to room temperature, suggests that the $\alpha 1-\alpha 2$ transition is a metastable-stable one. In the metastable state, chains are "frozen" in disorder as to "up or down arrangement." The transition is, in this meaning, a disorder-order one. The change in the X-ray pattern is analogous to that seen in the order-disorder transition of a metal alloy, though the temperature dependence is just opposite. The many new reflections ( $h k l$ with $h+k=$ odd) in the $\mathrm{X}$-ray pattern of the $\alpha 2$ form can be regarded as socalled super-lattice lines, which are illustrated in Figure 13.

\section{Energetic Considerations and the Crystallization Process}

The ipp molecular chain can be regarded as a coaxial column, the internal column consisting of the main chain and the external one consisting of the methyl-group helix. From energetic considerations, the interaction between the methyl-group helices would play the most important role in the interchain interactions. The interaction between the two helical chains in the isowinging is quite different from that in the antiwinding case. Compared with $\mathrm{CH}_{3}-$ $\mathrm{CH}_{3}$ distances, it is easily shown that much closer packing is realized in the antiwinding couple than in the isowinding couple. The packing and the distances are illustrated in Figure 14 and Table III, where the distance of the nearest neighbors is assumed to be $4.0 \AA$. This result agrees with our structural analysis that a helical chain is always surrounded by antiwinding helices in both the $\alpha 1$ and $\alpha$ forms. Thus it is reasonable that disorder with respect to "helical arrangement" would rarely occur on crystallization of an $\alpha$ form. The disorder with respect to "up or down arrangement," however, would not affect it so severely, because the effect caused by replacement of the internal columns would be less than the helical replace-

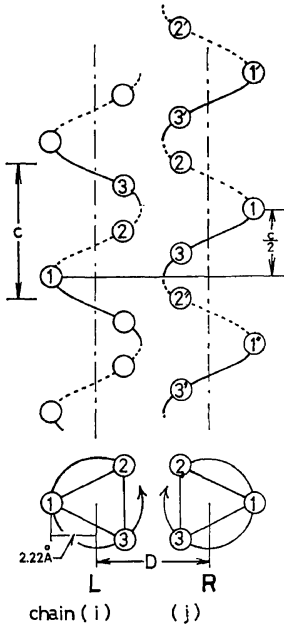

(a) antiwinding couple

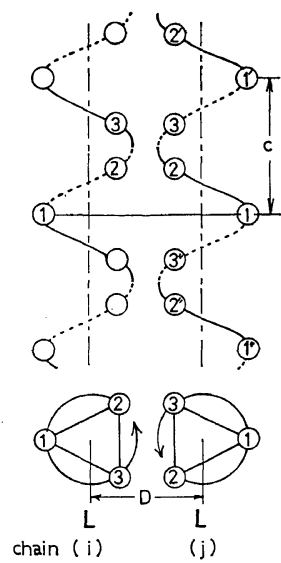

(b) iso winding couple

Figure 14. The closest packing of the two $\mathrm{CH}_{3}$ helical chains, assuming the distance between the nearest neighbors to be $4.0 \AA$. Here, $D=4.54 \AA$ for the anti-winding couple and $D=5.58 \AA$ for the iso-winding couple.

Table III. The $\mathrm{CH}_{3}-\mathrm{CH}_{3}$ distances $r_{i j}$ between $i$ - and $j$ - $\mathrm{CH}_{3}$-helices situated in the closest packing, shown in Figure 14, considering up to thirdnearest neighbors. $i^{\prime}, j^{\prime \prime}$, etc., are illustrated in Figure 14.

(a) Anti-winding case

\begin{tabular}{|c|c|}
\hline$i j$ & $r_{i j}(\AA)$ \\
\hline $22, \quad 22^{\prime \prime}, \quad 33, \quad 33^{\prime}$ & 4.00 \\
\hline $23, \quad 32$ & 4.63 \\
\hline $21, \quad 12, \quad 31, \quad 13$ & 6.07 \\
\hline
\end{tabular}

(b) Iso-winding case

\begin{tabular}{ccc}
\hline \multicolumn{2}{c}{$i j$} & $r_{i j}(\AA)$ \\
\hline 23, & 32 & 4.00 \\
22, & 33 & 5.11 \\
$32^{\prime}$ & & 5.49 \\
\hline
\end{tabular}

ment. But it is also reasonable that some energy difference is induced by the "up or down" replacement, which would cause the metastablestable transition.

\section{Diffuse Scattering in the Layer Lines}

In X-ray diagrams of oriented polymers, streaklike diffuse scattering often appears along layer lines. This type of diffuse scattering is especially 
striking with the isotactic ipp $\alpha$ form. Amano showed from observation at a low temperature (about $-100^{\circ} \mathrm{C}$ ) that the diffuse scattering in ipp was not due to the thermal lattice vibration but to structural defects in the crystal. ${ }^{6} \mathrm{He}$ insisted also from optical models that the defect was of the "block-type" in which many right repeating units were replaced in a row by wrong ones. If a unit cell containing "disorder" chains as to the up or down arrangement corresponds to a "wrong repeating unit," the intense streak in the $\alpha 1$ form and the decrease in intensity with increasing annealing temperature can be well interpreted.

\section{Disorder in ipp Solid}

The other disordered solid states of ipp have been studied by Natta $^{1}$ and Wyckoff. ${ }^{7}$ When a sample is melted and suddenly quenched in water, an extremely disordered state named smectic is obtained. ${ }^{1}$ Wyckoff proposed a three phases model: an amorphous, paracrystalline, and crystalline phase. According to this classification, the two phases, $\alpha 1$ and $\alpha 2$, belong to the crystalline phase. It would be possible to classify the phases in ipp crystal by use of the regularity as to chain arrangement, as is shown partly in this paper with respect to the $\alpha 1$ and $\alpha 2$ phases.

Ackowledginent. We wish to thank Mrs. I. Sakurai, Dr. S. Iwayanagi, and Dr. T. Sakurai of The Institute of Physical and Chemical Research for their helpful aid in calculating the structure factors by use of the program, RSSF3 of 5020 UNICS.

\section{REFERENCES}

1. G. Natta and P. Corradini, Suppl. Nuovo Cimento, 15, 40 (1960).

2. Z. Mencik, Chem. Prum., 10, 377 (1960).

3. A. Guinier, "Théorie et Technique de La Radiocrystallographie," K. Koora, S. Hosoyo, K. Doi, and N. Niiseki, Translators, Rigaku Denki Co., Ltd, Tokyo, 1967, Chapter 12.

4. M. Hikosaka and T. Seto, Rep. Progr. Polym. Phys. Jap., 12, 153 (1969).

5. M. Hikosaka and T. Seto, ibid., 14, 157 (1971).

6. T. Amano, Kolloid-Z., 206, 97 (1965).

7. H. W. Wyckoff, J. Polym. Sci., 62, 83 (1962).
Note Added in Proof. Z. Mencik reported results of a structural analysis of ipp based on $\mathrm{P} 21 / \mathrm{c}$ space group in J. Makromol. Sci. Phys., 136, 101 (1972). Chain shape and chain packing in his model are different from ours. The formula of the structure factor is also different. His formulation,

$$
F=c F_{\text {reg }}+(1-c) F_{\text {wrong }}
$$

where $F_{\text {reg }}, F_{\text {wrong, }}$ and $c$ are the structure factors of "order," "disorder" state as to up or down arrangement, and its volume fraction, demands a model in which the "disorder" chains are scattered randomly in a crystallite. This model can not give a reasonable interpretation for the change in the line width, because the widths of the reflections $h k l$ with $h+k=$ both even and odd similarly depend on the size of a crystallite, which contradicts the experimental facts. But if we use eq 14, his formula can be rewritten into an equivalent of eq 8 ,

$$
F=2(1-c) F_{\mathrm{C} 2 / \mathrm{c}}+(2 c-1) F_{\mathrm{P}_{1} / \mathrm{c}}
$$

The $R$-factor from Mencik is 0.158 , which is slightly larger than our value, 0.152 .

\section{APPENDIX}

We prove here that the superposition of two space groups $\mathrm{P} 2_{1} / \mathrm{c}(\uparrow)$ and $\mathrm{P} 2_{1} / \mathrm{c}(\downarrow)$ is equivalent to $\mathrm{C} 2 / \mathrm{c}$. Here, the coordinates of atom will be described in terms of the coordinate system of $\mathrm{C} 2 / \mathrm{c}$. The coordinates of an atom of $\mathrm{P} 2_{1} / \mathrm{c}(\uparrow)$, $(x, y, z)$, are transformed into those of $\mathrm{P} 2 / \mathrm{c}(\downarrow)$, $\left(x^{\prime}, y^{\prime}, z^{\prime}\right)$, by the relation

$$
\left(\begin{array}{l}
x^{\prime} \\
y^{\prime} \\
z^{\prime}
\end{array}\right)=\left(\begin{array}{rrr}
-1 & 0 & 0 \\
0 & 1 & 0 \\
0 & 0 & -1
\end{array}\right)\left(\begin{array}{l}
x \\
y \\
z
\end{array}\right)+\left(\begin{array}{l}
0 \\
0 \\
\frac{1}{2}
\end{array}\right)
$$

Using eq A1, we have eight coordinates of equivalent position, four of which are of $\mathrm{P} 2_{1} / \mathrm{c}(\uparrow)$ and the rest are of $\mathrm{P} 2_{1} / \mathrm{c}(\downarrow)$. They are

$$
\begin{aligned}
&\left\{\bar{x}, y, \bar{z}+\frac{1}{2} ; x+\frac{1}{2}, \bar{y}+\frac{1}{2}, z+\frac{1}{2} ;\right. \\
&\left.x+\frac{1}{2}, y+\frac{1}{2}, z ; \bar{x}, \bar{y}, \bar{z}\right\}
\end{aligned}
$$

and 
The Order of the Chains in Ipp Crystals

$$
\begin{aligned}
& \left\{x, y, z ; \bar{x}-\frac{1}{2}, \bar{y}+\frac{1}{2}, \bar{z}\right. \\
& F_{\mathrm{P}_{1} / \mathrm{c}}(\uparrow)+F_{\mathrm{P}_{1} / \mathrm{c}}(\downarrow)=2 F_{\mathrm{C} 2 / \mathrm{c}} \\
& \left.\bar{x}-\frac{1}{2}, y+\frac{1}{2}, z+\frac{1}{2}, x, y, z+\frac{1}{2}\right\} \text { (A2) where } F_{\mathrm{C} 2 / \mathrm{c}} \text { is taken as eq } 7 \text {. Eq A3 is the }
\end{aligned}
$$

These coincide with the equivalent positions of same as eq 14 .

the $\mathrm{C} 2 / \mathrm{c}$ space group. Thus we have 